DOI: 10.25100/eg.v0i21.11294 Espacios y Territorios

\title{
Inventario y composición florística de la flora urbana de uso ornamental y paisajístico en el municipio de Peque (Occidente de Antioquia)
}

\section{Inventory and floristic composition of urban flora for ornamental and landscape use in Peque town (West of Antioquia)}

\author{
José Ubeimar Arango Arroyave \\ Magíster en Medio Ambiente. Profesional Independiente. Colombia. \\ egoronomia@hotmail.com | 0000-0002-1574-5705
}

\begin{abstract}
Para citar este artículo: Arango Arroyave, J. U. (2021). Inventario y composición florística de la flora urbana de uso ornamental y paisajístico en el municipio de Peque (Occidente de Antioquia). Entorno Geográfico, (21), 77-105. https://doi.org/10.25100/eg.v0i21.11294
\end{abstract}

\section{Resumen}

El presente trabajo muestra tanto el inventario como la composición florística de la vegetación de uso ornamental más representativa del área urbana del municipio de Peque a nivel silvicultural, de zonas verdes y de embellecimiento paisajístico, ya que dentro su perímetro urbano y semiurbano conserva, combina, gestiona y mantiene e integra su cobertura vegetal en sus procesos de ordenamiento territorial. Para esto se partió de técnicas de investigación cuantitativas basadas en el cálculo de abundancia absoluta y relativas, índices de diversidad a nivel de Shannon, Índice de Berger- Parker y Cociente de mezcla, las cuales se complementaron con técnicas de tipo cualitativo a nivel de sondeos rurales rápidos y participativos, talleres, registro audiovisual (fotografía y video), entrevistas semiestructuradas, observación directa y participante; además se realizó muestreo para la toma de ejemplares botánicos, formatos, notas y recorrido de campo para la posterior identificación taxonómica; donde se reportan 56 familias, 97 géneros, 107 especies y 781 individuos, de las cuales sobresalen en mayor cantidad 39 familias que hacen parte del grupo Angiospermae subclase Magnoliopsida (Dicotiledoneae), con 78 especies, seguido por la subclase Liliopsida (Monocotiledoneae) con 13 familias y 24 especies; en orden descendente aparecen las Gymnospermas (coníferas y afines) con 3 familias y 4 especies y por último el grupo de las Pteridofitas (Helechos) con 1 familia y 1 especie respectivamente, con el fin de que se convierta 
en un aporte al conocimiento y valoración de una parte de la flora local y como punto de referencia en la toma de decisiones y de planificación frente a su manejo.

Palabras clave: Arborización urbana, Biodiversidad vegetal, Zonas verdes, Occidente de Antioquia.

\begin{abstract}
This paper makes an inventory and the floristic composition vegetation ornamental use most representative of the urban sites of Peque town at the silvicultural level, Gardening and landscaping beautification, since within its urban and semi-urban perimeter it preserves, combines, manages and maintains and integrates its vegetation cover in its land use planning processes. It started with quantitative research techniques based on the calculation of absolute and relative abundance,

diversity indices at the Shannon level, Berger-Parker index and Mixing ratio, which were complemented with qualitative techniques at the sounding level. fast and participatory rural activities, workshops, audiovisual recordings (photography and video), semi-structured interviews, direct and participant observation; In addition, sampling was carried out to take botanical samples, formats, notes and a field trip for subsequent taxonomic identification; where 56 families, 97 genera and 107 species are reported, of which 39 botanical families that form part of the Angiospermae group subclass Magnoliopsidae (Dicotiledoneae) stand out in greater quantity, with 78 species, followed by the subclass Liliopsidae (Monocotiledoneae) with 13 botanical families and 24 species, in descending order appear the Gymosperms (coniferous and related) with 3 botanical families and 4 species and finally the group of Pteridophytes (Ferns) with 1 family and 1 species respectively, in order to become a contribution to knowledge and valuation of a part of the local flora and as a reference point in decision-making and planning regarding its management.
\end{abstract}

Keywords: Green areas, Plant biodiversity, Urban tree planting, West of Antioquia

Recibido: 06 de agosto de 2020

Aceptado: 23 de septiembre del 2020

\title{
Introducción
}

La flora urbana prolonga lo verde dentro de lo artificial, haciendo de lo urbano un lugar más agradable y estético, desempeñando funciones que van más allá de lo meramente paisajístico, cumpliendo con una serie de interacciones ecológicas y servicios ambientales como la captura de emisiones de gases de efecto invernadero, control de la erosión, regulación de las aguas lluvias, micro regulación climática urbana a nivel de la temperatura y del aire, reducción de ruido y 
albergue de flora epífita como de fauna (Costanza et al., 1997; Amaya 2005; Morales y Varón, 2006). También puede ayudar al mantenimiento de los valores socioculturales de los bosques en la planificación urbanística con otros beneficios como la promoción de productos forestales locales y el turismo, así como el aumento de la calidad de vida, como del bienestar físico y mental de los habitantes de las localidades y la garantía de que las generaciones actuales y venideras se beneficien de una variedad de paisajes culturales y naturales que contribuyan en alcanzar uno de los Objetivos de Desarrollo Sostenible (ODS) concretamente en el número 11 que habla de "proteger y salvaguardar el patrimonio cultural y natural del mundo" (Salbitano et al., 2017).

Es así como estos mismos autores definen los bosques urbanos: Como redes o sistemas que comprenden todos los arbolados (rodales), grupos de árboles y árboles individuales ubicados en las áreas urbanas y periurbanas; por tanto, se incluyen bosques, árboles en las calles, árboles en los parques y jardines y árboles en las esquinas de las calles. Los bosques urbanos son la espina dorsal de la infraestructura verde que conecta las áreas urbanas a las rurales y mejora la huella ambiental de las ciudades.

Estos mismos autores continúan en su definición incorporando el concepto de forestería urbana y periurbana para referirse al arbolado urbano, como la práctica de gestión de los bosques urbanos para asegurar su contribución óptima al bienestar psicológico, sociológico y económico de las sociedades urbanas. Es un enfoque integrado, interdisciplinario, participativo y estratégico de planificación y gestión de los bosques y árboles en y alrededor de las ciudades. Engloba la evaluación, planificación, plantación, mantenimiento, preservación y monitoreo de los bosques urbanos y puede operar a escalas que van desde un solo árbol hasta todo el paisaje.

Este tipo de arborización a manera de zonas verdes o jardines domésticos viene cobrando importancia dentro de las áreas urbanas ocupando notables superficies incluso en zonas residenciales dispersas (Gaston et al., 2005; Loram et al., 2007; Mathieu et al., 2007), en el cual el área de estudio en mención no es la excepción, evidenciando la importancia de este tipo de cubiertas vegetales dentro del uso del suelo urbano ligados no solamente a aspectos y factores de orden natural sino también socioeconómico (Padullés et al, 2015). Sin desconocer además la importancia que vienen teniendo las coberturas arbóreas sobre procesos de planeación e infraestructura urbano regional (Howes y Herbert, 2005). 
No obstante es importante señalar como lo menciona (Tovar- Corzo, 2013) de que a silvicultura urbana en algunos países latinoamericanos como es el caso también de Colombia se ha visto simplificada desde la época de la conquista y la colonia y no fue hasta hace más o menos 20 años, que se han fortalecido de manera institucional, normativa y participativa las principales ciudades en el país como lo son Bogotá, Medellín y Cali en donde hay avances significativos en cuanto a protocolos y manuales técnicos asociados a silvicultura y manejo de arbolado urbano, censos forestales georeferenciados e información institucional importantes para la gestión del arbolado urbano. Entre tanto ciudades como Cartagena de Indias, Pasto y Cúcuta se encuentran en proceso de elaboración de censo de arbolado urbano que van articulados con proyectos de impacto urbano.

En esta misma línea enunciada anteriormente, el presente trabajo pretende contribuir mediante la identificación, estructura, composición, inventario de la biodiversidad del arboretum representada en la flora de uso ornamental y paisajística en el municipio de Peque, que faciliten procesos y planes de manejo de estos ecosistemas urbanos, constituyendo una línea base y de planificación a futuro para la toma de decisiones frente a los usos del suelo en lo referente a sus coberturas vegetales y se apoyó en varios parámetros y metodologías brindadas por la silvicultura de bosques

y la ecología forestal, la cual se adaptó y viene siendo adaptada en varios trabajos actuales al estudio de la composición y estructura de los bosques y coberturas vegetales urbanas, las cuales han sido recopiladas por autores como (Magurran, 1988; Moreno, 2001 y Melo y Vargas, 2001)

\section{Materiales y Métodos}

\section{Área de estudio y características}

El Municipio de Peque, se ubica en el extremo Noroccidental del departamento de Antioquia, en las coordenadas $7^{0} 01$ ' 13 '’ $\mathrm{N}$ y 750054'18'’O, con una extensión de $392 \mathrm{Kms}^{2}$, dista 239 kilómetros de la ciudad de Medellín (Figura 1); se diferencia de los demás municipios del Occidente antioqueño ya que su territorio se dispone hacia la cuenca del Río Cauca (CORPOURABA,2009). Cartográficamente, la cabecera Municipal se encuentra a 1200 metros sobre el nivel del mar, (IGAC, 2007), correspondiente a la zona de Vida de Bosque Seco Tropical (bs-T), con límites climáticos generales de biotemperaturas superiores a $24^{\circ} \mathrm{C}$ y precipitaciones entre los 1.000 y 2.000 mm promedio anual (Espinal, 1992; citado por Idárraga et al, 2011).

Los suelos se caracterizan según (IGAC, 2007), por su poca profundidad efectiva exceptuando los que se encuentran en la zona cafetera que tienden a ser moderadamente profundos con susceptibilidad a la erosión, que varía de ligera a severa, estado de fertilidad natural de media a 
baja y un relieve quebrado a escarpado. Las vertientes que rodean el casco urbano muestran áreas en caficultura, (Benítez, 1997; CORPOURABA, 2009).

Fuente: Oficina de catastro municipio de Peque Julio de 2020.

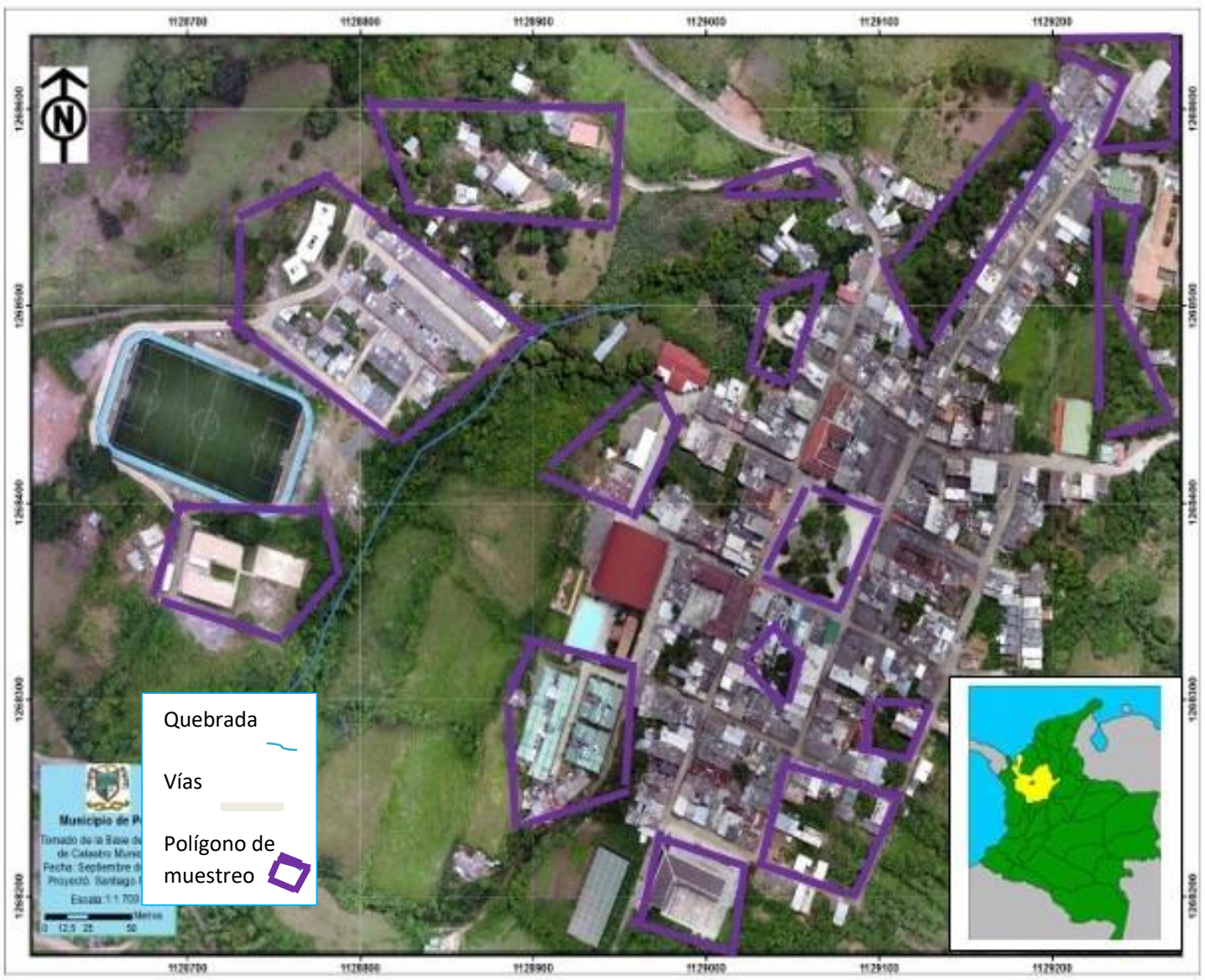

Figura 1. localización de la zona de estudio con su respectiva cobertura vegetal circundante y áreas de muestreo (Polígonos de color morado).

El proceso metodológico se basó en dos fases una de campo y otra de oficina las cuáles se describen a continuación:

Trabajo de campo: Esta fase contó con 18 entrevistas semi-estructuradas realizadas a expertos (Albuquerque et al.,2014) y 8 recorridos de campo con énfasis en aspectos etnobotánicos (Medeiros et al., 2014), las 36 personas entrevistadas corresponden a 24 estudiantes de la media técnica en manejo ambiental del proceso de articulación SENA- I.E Presbítero Rodrigo Lopera Gil, cuatro empleados de la UMATA (Unidad Municipal de Asistencia Técnica Agropecuaria), uno de la oficina de CORPOURABA (Corporación para el Desarrollo Sostenible del Urabá) en Peque, uno de la oficina de planeación Municipal, el director del Parque Educativo "La Verdadera Capital 
de la Montaña"; dos empleados de la Empresa de Servicios Públicos del municipio y cuatro botánicos tradicionales que habitan en el casco urbano del municipio.

Esto se complementó con notas, registros fotográficos y de campo, videos, listas con datos para posterior trabajo de herbario, visitas a los sitios de muestreo donde se encuentran los espacios más representativos a nivel de zonas verdes, parques y barrios dentro del perímetro urbano seleccionando como sitios de muestreo con sus respectiva área : El Parque principal $\left(3600 \mathrm{~m}^{2}\right)$, el parque Educativo $\left(180 \mathrm{~m}^{2}\right)$, alrededores del cementerio $\left(4800 \mathrm{~m}^{2}\right)$, zona verde del Hospital San Francisco de Asís" $\left(400 \mathrm{~m}^{2}\right)$, sector de la virgen en el barrio el Cielito $\left(35 \mathrm{~m}^{2}\right)$, zona verde de la urbanización los mangos $\left(2000 \mathrm{~m}^{2}\right)$, la Máquina $\left(300 \mathrm{~m}^{2}\right)$, alrededores de la I.E Presbítero Rodrigo Lopera Gil $\left(96 \mathrm{~m}^{2}\right)$ todos pertenecientes al barrio la Miranda, sector verde del barrio Italia Noventa $\left(150 \mathrm{~m}^{2}\right)$, urbanización Valle del Tesoro $\left(4000 \mathrm{~m}^{2}\right)$, Casa de la Cultura $\left(60 \mathrm{~m}^{2}\right)$, Fundungo $\left(100 \mathrm{~m}^{2}\right)$, zona verde barrio Villa Nueva $\left(300 \mathrm{~m}^{2}\right)$; sector el Caliche $\left(200 \mathrm{~m}^{2}\right)$, el Condorcito $\left(80 \mathrm{~m}^{2}\right)$ y zona verde salida hacia el Tornillo $\left(160 \mathrm{~m}^{2}\right)$ ver Figura 1 . También se dio espacio a aspectos de observación directa y participante (Sánchez, 2004); así como de conversaciones de tipo informal (Devillard et al.,2012).

Trabajo de oficina: En esta fase se procedió a identificación botánica y composición florística del material vegetal muestreado; se procedió para la clase Liliopsidae (Monocotiledoneae) el sistema propuesto por (Dahlgren et al., 1985); para la clase Magnoliopsidae (Dicotiledoneae) el de (Cronquist, 1988), procediendo además a consultar la base de datos del Jardín Botánico de Missouri; así como la base de datos del catálogo de especies de la flora urbana del Valle de Aburrá de la Escuela de Ingeniería de Antioquia

En cuanto a la diversidad florística se calculó:

Abundancia absoluta: Se refiere al número de individuos pertenecientes a una determinada especie presente en una muestra. (Lamprecht, 1990, citado por Cantillo y Gracia, 2013)

Abundancia relativa: Es el porcentaje de individuos de una especie con respecto al número total de individuos de la muestra (Lamprecht, 1990, citado por Cantillo y Gracia, 2013).

$\mathrm{Ar}=\mathrm{Ni} / \mathrm{N}^{* 100}$ Dónde: $\mathrm{Ar}=$ abundancia relativa $\mathrm{Ni}=$ número de individuos de la especie $\mathrm{N}=$ número de individuos de la muestra 
Índice de Riqueza o Cociente de mezcla (C.M), el cual consiste en la razón entre el número de individuos y el correspondiente número de especies (Moreno, 2001); el cual se calcula como: C.M. $=$ N/S Dónde: $\mathrm{C}: \mathrm{M}:=$ Cociente de Mezcla $\mathrm{N}=$ Número total de individuos de la muestra $\mathrm{S}=$ Número total de especies de la muestra

Índice de Shannon-Weiner, índice que refleja la relación entre riqueza y uniformidad, (Moreno, 2001), definiéndose como:

$H^{\prime}=$ - $(P i) x \operatorname{Ln}(P i)$ donde: $\mathrm{H}^{\prime}=$ Índice de Shannon- Weiner Pi = ni/N Proporción de individuos de la $\mathrm{i}$-ésima especie $\mathrm{Ni}=\mathrm{N}^{\circ}$ de individuos de la $\mathrm{i}$-ésima especie.

Índice de Berger- Parker (D): Es un índice de dominancia que varía entre 0 y 1, cuanto más se acerca a 1 significa que mayor es la dominancia y menor la diversidad. $\mathrm{D}=\mathrm{Nmáx} / \mathrm{N}$ Donde: Nmáx = abundancia de la especie dominante (Moreno, 2001).

Esto se complementó con el análisis y síntesis de la información etnobotánica recopilada; fuentes de información secundaria proveniente de la sección de las hemerotecas de la biblioteca "Efe Gómez" de la Universidad Nacional de Colombia sede Medellín y de la Universidad de Antioquia “Carlos Gaviria Díaz” y de sus respectivos sistemas repositorios digitales de estas dos bibliotecas, así como del repositorio virtual de la UNAD (Universidad Nacional Abierta y a Distancia).

\section{Resultados y Discusión}

La composición florística del inventario realizado sobre flora de uso ornamental y paisajístico localizada dentro del perímetro urbano y sus alrededores está representada por 56 familias, 97 géneros y 107 especies, de las cuales sobresalen en mayor cantidad 39 familias que hacen parte del grupo Angiospermae subclase Magnoliopsida (Dicotiledoneae), con 78 especies, seguido por la subclase Liliopsida (Monocotiledoneae) con 13 familias y 24 especies, en orden descendente aparecen las Gymnospermas (coníferas y afines) con 3 familias y 4 especies y por último el grupo de las Pteridofitas (Helechos) con 1 familia y 1 especie respectivamente (ver Anexo 1). El inventario realizado reporta 781 individuos, siendo las especies más abundantes y dominantes en su orden El maní forrajero (Arachis pintoi Krap et Greg) con 400 individuos, seguido de las durantas (Duranta spp L) con 80, Sunglia llamado también como limón ornamental (Swinglea glutinosa (Blanco) Merr) con 25, Nogal cafetero (Cordia alliodora (Ruiz \& Pav.) Cham) y palma manila (Veitchia merrillii (Becc) H. Moore) con 16 individuos respectivamente, acalifa (Acalypha 
amentaceae f. circinata (Müll.Arg.) Fosberg) con 12, las restantes especies se encuentran entre 1 y 9 (ver Anexo 2).

En trabajos similares y a nivel comparativo, aunque con algunas diferencias (Palacio y Pedraza, 2012) reportan para las zonas verdes inventariadas del Politécnico Jaime Isaza Cadavid en la Ciudad de Medellín una composición florística de 26 familias, 45 géneros, 49 especies y 566 individuos. Representado respectivamente y en orden de abundancia las especies Eucalipto (Eucalyptus sp), Tulipánes africanos (Spathodea campanulata P. Vaud), Rapones (Fainas chínense (Lenz) Ringles), todas ellas especies introducidas.

Para este trabajo de las 107 especies identificadas, 7 con un $6.54 \%$ hacen parte de la flora nativa y dejadas dentro del espacio urbano con fines ornamentales y paisajísticos representadas por la ceiba (Ceiba pentandra (L) Gaertn), piñón de oreja (Enterolobium cyclocarpum (Jacq.) Griseb), indio desnudo (Bursera simaruba (L) Sarg), yarumo (Cecropia spp Loefl), chumbinbo (Sapindus saponaria L), aguacatillo (Persea caerulea (R\&P) Mez) y guadua (Guadua angustifolia kunth), las restantes 100 han sido introducidas, ya sea por propuestas de intervención urbanística en diferentes momentos, como por iniciativa de algunas personas que las siembran por aspectos estéticos que de algunas de ellas emanan. En este sentido es importante mencionar como esto conlleva a establecer una serie de percepciones y preferencias por los espacios y zonas verdes que tienden a diferir debido a una serie de variables tanto de los moradores del lugar como de quienes los visitan.( Ozguner y Kendle, 2006; Schipperijn et al., 2010; Wan y Shen, 2015; R. Wang, Zhao, y Liu , 2016; Zhang, Yang, Ma y Huang, 2015) y que se encuentran en función de variables como: La edad, el género, el estatus migratorio y los antecedentes culturales (Farahani y Maller, 2018); bajo esta perspectiva un entorno determinado tienda a ser experimentado de diferentes formas y maneras por diferentes personas y es a través de estas múltiples experiencias y transformaciones de un espacio a otro que las zonas verdes urbanas adquieren significado y múltiples valores (Qiu y Nielsen, 2015).

Con respecto a los hábitos de crecimiento se tienen de mayor a menor 31 especies son árboles (29\%), 30 Herbáceas (28\%), 27 arbustos (25\%), 8 son hierbas gigantes (7\%), 6 sub arbustos (6\%), 3 palmas (3\%) y 2 bejucos (2\%). Ver Anexo 1 y Figuras 2 y 3. La diversidad biológica y morfológica encontrada acá permite una mejor utilización de los recursos y del espacio. Al respecto (Altieri, 1993) comenta que el agrupamiento de plantas de diferentes hábitos de crecimiento, con follajes y estructuras radiculares distintas, permite una mejor utilización de los factores 
ambientales, tales como los nutrientes, el agua y la radiación solar, planteando además, que la diversidad de especies, su distribución y arreglos disminuye notablemente la presencia y la actividad de las plagas y enfermedades y facilita la supervivencia de enemigos naturales, disminuyendo el uso de agroquímicos, el uso de energía externa y por tanto, los costos de manejo de prácticas tipo agrosilvocultural.

Fuente: Elaboración propia.

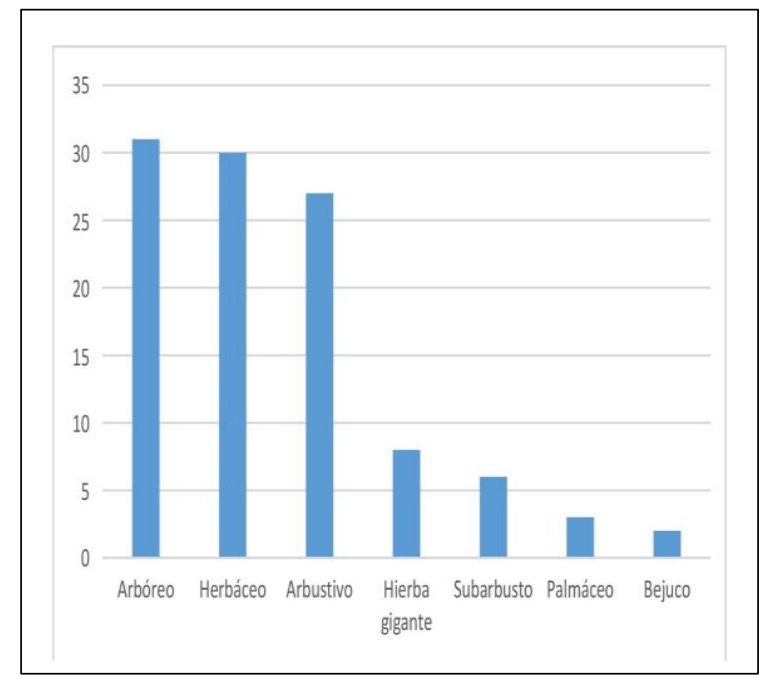

Figura 2. Número de especies encontradas por hábitos de crecimiento de acuerdo al área de muestreo.

Fuente: Elaboración propia

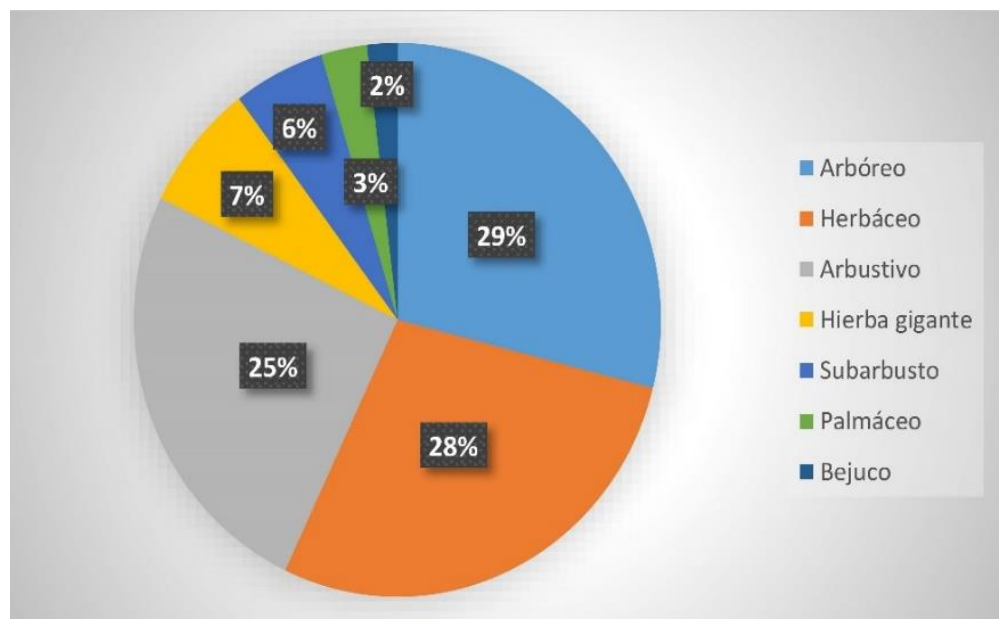

Figura 3. Proporción de hábitos de crecimiento correspondiente al área de muestreo. 
En cuanto a los índices de diversidad encontrados para el presente trabajo se tiene un Índice de Riqueza o Cociente de mezcla de 7.2656 y de 2.5917 para el Índice de Shannon ver Anexo 2, el primero indica la probabilidad de que un individuo se encuentre en una muestra tomada al azar y el segundo refleja la relación entre riqueza y uniformidad (Magurran, 1988; citado por Moreno, 2001), el valor calculado para el casco urbano de Peque, indica que la diversidad es relativamente baja a media. Para el Índice de Berger- Parker el valor encontrado fue de 0.5121 ver Anexo 2, ya que su valor se encuentra entre 0 y 1 , si el valor tiende a acercarse a ser mayor indica un aumento en la equidad y una disminución en la dominancia, un valor bajo significa que no hay una especie marcadamente dominante. Magurran (1988, citado por Moreno, 2001) que corresponde para este caso con la máxima abundancia relativa encontrada para la especie Arachis pintoi. Con respecto a la medición de índices de diversidad en trabajos similares a este, (Palacio y Pedraza, 2012) reportan para las zonas verdes inventariadas del Politécnico Colombiano "Jaime Isaza Cadavid" en la Ciudad de Medellín para Shannon-Weiner 2.5467 y para Berger-Parker de 0.21, lo cual permite hacer comparaciones encontrando una pequeña diferencia con respecto al primero, siendo más marcada para el segundo.

Más allá de los valores encontrados en este trabajo en términos de una menor o mayor diversidad hace que no sea fácil realizar comparaciones y análisis con masas forestales y boscosas solamente influenciadas por procesos ambientales, ecológicos y de selección natural; dado que el ecosistema analizado acá corresponde más a un ecosistema urbano con una alto grado de intervención antrópica, por lo que los valores reportados acá están en función de otras variables como gustos, preferencias en la selección y siembra de especies y percepciones estéticas de quienes van enriqueciendo estos espacios verdes.

Con respecto a las preferencias humanas estas según (Padullés et al., 2015) ejercen una fuerte presión de selección sobre el número y el tipo de especies exóticas introducidas en los hábitats urbanos, así como también en la forma en que éstas son gestionadas (Hope et al., 2003; Luck et $a l ., 2009)$. La probabilidad de que se establezca una nueva población se encuentra directamente relacionada con la incorporación del número de individuos con capacidad reproductiva. En este sentido, la preferencia humana actúa como filtro evidente favoreciendo algunas especies por encima de otras. (Williams et al. 2009) concluye que tales preferencias comportan un aumento neto del número de especies del sistema; En este misma línea y perspectiva de análisis (Halffter y Ezcurra, 1992) como (Escobar, 1997) hacen referencia en que la biodiversidad es el resultado de la interacción entre el ecosistema y la cultura. 
Esta biodiversidad representada en el arboretum urbano identificado y reconocido localmente en el presente trabajo genera desde el punto de vista paisajístico y estético un ambiente visualmente agradable, dada la forma como están distribuidas las especies y los individuos en el contorno urbano del municipio de Peque, en este sentido siguiendo a (Salbitano et al., 2017), los bosques urbanos, bien diseñados y distribuidos, pueden tener funciones claves para aumentar la igualdad social, promover un sentido de comunidad entre los residentes y garantizar el mantenimiento de los valores culturales locales.

Dentro de las representaciones sociales de la flora inventariada y muestreada se encuentran en el Parque principal, caracolí (Anacardium excelsum (Bert \&Balb. Ex Kunth) Skeels), el cual fue sembrado con una placa conmemorativa a la vida, conocido y bautizado como el "Árbol de la vida" haciendo alusión a querer pasar la página de la violencia y conflicto armado que se vivió muy fuertemente hace unos años atrás en la zona y el mango (Mangifera indica), que de acuerdo a relatos surgidos de las entrevistas realizadas las personas concuerdan que donde está sembrado fue el sitio en el cual los fundadores del municipio se toparon con dos osos de anteojos (Tremarctos ornatus).

De la flora encontrada acá 10 especies hacen alusión a emblemas y símbolos de algunos países (Morales y Varón, 2006) como el caso de Roble (Tabebuia rosea (Bertol) A.DC- árbol nacional de Salvador), Guayacán amarillo, (Tabebuia chrysantha (Jacq) Nicholson - árbol nacional de Venezuela), Piñón de oreja (Enterolobium cyclocarpum (Jacq.) Griseb- árbol nacional de Costa Rica), San Joaquín (Hibiscus rosa-sinensis L- árbol nacional de Indonesia), Clavellino árbol nacional de Barbados, Cresta de gallo (Erythrina crista-galli L- árbol nacional de Argentina y Uruguay), Ceiba (Ceiba pentandra (L) Gaertn- árbol nacional de Guatemala y Puerto Rico). Eucalipto (Eucalyptus spp - árbol nacional de Australia), Curazao (Bougainvillea glabra Choisyárbol nacional de Aruba y Curazao) y Araucaria (Araucaria excelsa R.Br - árbol nacional de Chile).

En sí la flora ornamental que hace parte de la cobertura urbana del municipio es relativamente joven siendo en su mayoría especies introducidas y establecidas en gran parte desde finales de los años ochenta, renovándose y enriqueciéndose con individuos joviales como sucede en espacios como el parque educativo, el parque principal y el sector del barrio La Miranda desde el año 2015. Sin embargo, en algunos individuos del componente arbóreo sobre todo del parque principal se requiere de podas y de retiro de algunas especies epífitas del género Tillandsia conocidas como melenas y golondrinas, aunque sin ser parasitarias dan un aspecto no muy deseado estéticamente. 
Desde el punto de vista de sanidad vegetal a pesar de no encontrarse problemas fitosanitarios de alta magnitud se ven ataques esporádicos de hormigas arrieras del género Atta y ataques de insectos de la familia Chrysomelidae sobre todo en durantas como de fumagina en algunos de los ornamentales. En el parque principal se hace necesario pensar en el reemplazo de un individuo de la especie ya que presenta un grave deterioro en su fuste que lo hace de alto riesgo por una posible caída.

\section{Conclusiones}

El conocimiento e identificación de la flora con énfasis en su uso como parte del embellecimiento paisajístico en entornos urbanos como el presentado en el siguiente trabajo puede ser utilizado en el afianzamiento de los procesos que se vienen llevando en torno a la planificación, diseño y gestión de los espacios verdes, posibilitando que las comunidades y moradores fortalezcan lazos de identidad, apropiación y valores socioculturales con su entorno ambiental y geográfico circundante.

Este tipo de trabajos puede generar también más adelante estudios o líneas de investigación en torno a la valoración, cuantificación e importancia de las coberturas vegetales urbanas frente a la mitigación del cambio climático, la huella ecológica, como sumideros de carbono, identificación de los servicios ecosistémicos prestados en los ambientes urbanos y periurbanos y en estudios socioculturales en términos de patrimonio ambiental y cultural y en estudios de interconexión de la flora urbana con criterios de calidad de vida y de salud de sus habitantes.

Si bien externa y aparentemente la flora urbana y periurbana de los espacios muestreados presenta condiciones de salud representadas en su estado fitosanitario relativamente en buenas condiciones se hace importante la realización de estudios detallados de sanidad vegetal que hagan posible una mejor toma de decisiones frente a la renovación, reposición y retiro de algunos individuos y especies que estén generando riesgos a la infraestructura, que permitan además posibles trámites y permisos ante las autoridades ambientales de la zona en cuanto a la tala, poda y trasplante cuando sea requerido.

\section{Agradecimientos}

Dentro del proceso, desarrollo y culminación de esta investigación se hace extensivo los agradecimientos institucionales al SENA en su Complejo Tecnológico, Turístico y Agroindustrial del Occidente Antioqueño, como a la Institución Educativa Presbítero Rodrigo Lopera Gil, sin dejar a nivel personal de dar las gracias a Maira Ruíz Asesora PRAES, a Tobías de los Milagros Arboleda Jaramillo ex Rector de la I.E Rodrigo Lopera Gil como Diego Zapata curador del 
Herbario Forestal de la Universidad Distrital "Francisco José de Caldas". Holver Arango Álvarez por la revisión del texto. A todos ellos por el aporte, asesoría y ánimo de dar a conocer este trabajo.

\section{Referencias bibliográficas}

Albuquerque U. P., Fernandes, L. V., Paiva, R. F., y Nóbrega, R. (2014). Methods and Techniques in Ethnobiology and Ethnoecology. England: Humana Press.

Allaby, M. (1992). The Concise Oxford dictionary of botany. Oxford, England: Oxford University Press.

Altieri, M. (1993). ¿Por qué estudiar la agricultura tradicional? En Curso sobre agroecología. Módulo I: Agroecología: Bases Históricas y teóricas (pp. 71-81). Santiago de Chile: CLADES.

Alzate, F., Idárraga. A., Díaz. O., y Rodríguez, W. (2013). Flora de los bosques montanos de Medellín. Medellín, Colombia: Señal Gráfica Impresión. Alcaldía de Medellín y Universidad de Antioquia.

Amaya, C. (2005). El ecosistema urbano: simbiosis espacial entre lo natural y lo artificial. Revista forestal latinoamericana, 20(37), 1-16.

Benítez, D. (1997). Estudio florístico y algunos elementos estadísticos aplicados a la técnica RAP en bosques relictuales del municipio de Peque-Antioquia (Trabajo de grado). Facultad de Ciencias Agropecuarias, Departamento de Ciencias Forestales, Universidad Nacional de Colombia Medellín.

Cantillo, E., y Gracia, M. (2013). Diversidad y Caracterización Florística de la Vegetación Natural en tres sitios de los Cerros Orientales de Bogotá D.C. Colombia Forestal, 16(2), 228-256. https://bit.ly/3taNoZn

Costanza, R., D’arge, R., De groot, R., Farber, S., Grasso, M., Hannon, B., Limburg, K., Naeem, S., O’Neill, R., Paruelo, J., Raskin, R., Sutton, P., y Van den belt, M. (1997). The value of the world's ecosystem services and natural capital. Nature, 387, 253-260. https://doi.org/10.1038/387253a0

Corporación para el Desarrollo Sostenible del Urabá. (2009). Plan de ordenamiento y manejo de la cuenca hidrográfica del río Peque. Apartadó, Colombia.

Cronquist, A. (1988). The evolution and classification of flowering plants. New York: The New York Botanical Garden.

Dahlgren, R. M. T., Clifford, H. T., y Yeo, P. F. (1985). The Families of the Monocotyledons. Structure, evolution and taxonomy. Berlin: Springer-Verlag.

Devillard, M. J, Franzé-Mudanó, A., y Pazos, Á. (2012). Apuntes metodológicos sobre la conversación en el trabajo etnográfico. Política y Sociedad,49(2), 353-369. https://doi.org/10.5209/rev_POSO.2012.v49.n2.36512 
Escobar, A. (1997). El final del salvaje: antropología y nuevas tecnologías. Santafé de Bogotá: Centro de Estudios de la Realidad Colombiana, Instituto Colombiano de Antropología, Ministerio de Cultura.

Espinal, L. (1992). Geografía Ecológica de Antioquia basadas en Zonas de Vida. Medellín: Editorial Lealon.

Farahani, L. M., y Maller, C. (2018). Perceptions and Preferences of Urban Greenspaces: A Literature Review and Framework for Policy and Practice. Lanscape ecology, 61, 1-22. https://doi.org/10.3097/LO.201861

Gaston, K. J., Warren, P. H., Thompson, K., y Smith, R.M. (2005). Urban domestic gardens (IV): the extent of the resource and its associated features. Biodiversity and Conservation, 14(14), 3327-3349. https://doi.org/10.1007/s10531-004-9513-9

Halffter, G., y Ezcurra, E. (1992). ¿Qué es la biodiversidad? La diversidad biológica en Iberoamérica I. Acta Zoología Mexicana, 24(2), 11-29.

Hope, D., Gries, C., Zhu, W. X., Fagan, W. F., Redman, C. L., Grimm, N. B., Nelson, A. L., Martin, C., y Kinzig, A. (2003). Socioeconomics drive urban plant diversity. Proceedings of the National Academy of Sciences of the United States of America, 100(15), 8788-8792. https://doi.org/10.1073/pnas.1537557100

Howes, R., y Herbert, R. (2005). Infrastructure for the Built Environment: Global procurement strategies. Oxford, Butterworth-Heinemann.

Idárraga, P., A., y R. Callejas P. (2011). Análisis florístico de la vegetación del Departamento de Antioquia. En A. Idárraga, R. del C. Ortiz, R. Callejas y M. Merello (Eds.), Flora de Antioquia: catálogo de las plantasvasculares. Vol. II. Listado de las plantas vasculares del departamento de Antioquia. Programa Expedición Antioquia-2020. Series Biodiversidad y Recursos Naturales. Bogotá, Colombia: Universidad de Antioquia, Missouri Botanical Garden \& Oficina de Planeación Departamental de la Gobernación de Antioquia, Editorial D’Vinni,

Instituto Geográfico Agustín Codazzi. (2007). Mapa de Suelos. Plancha 115, escala 1:100000. Bogotá, Colombia.

Loram, A., Tratalos, J., Warren, P. H., y Gaston, K. J. (2007). Urban domestic gardens (X): the extent \& structure of the resource in five major cities». Landscape Ecology, 22(4), 601-615. https://doi.org/10.1007/s10980-006-9051-9

Luck, G. W., Smallbone, L. T., y O'brien, R. (2009). Socio-economics and vegetation change in urban ecosystems: Patterns in space and time. Ecosystems, 12, 604-620. https://doi.org/10.1007/s10021-009-9244-6

Magurran A. E. (1988). Why diversity? En Ecological Diversity and Its Measurement. Springer, Dordrecht. https://doi.org/10.1007/978-94-015-7358-0_1

Mathieu, R., Freeman, C., y AryaL, J. (2007). Mapping private gardens in urban areas using object oriented techniques and very high-resolution satellite imagery. Landscape and Urban Planning, 81(3), 179-192. https://doi.org/10.1016/j.landurbplan.2006.11.009 
Medeiros, P. M., Almeida, A. L., Lucena, R. F., Souto, F. B., y Albuquerque, U. P. (2014). Use of Visual Stimuli in Ethnobiological Research. En U. L. Albuquerque, R. Fernandez, R. Farias, y R. Nóbrega (Eds.). Methods and Techniques in Ethnobiology and Ethnoecology. England: Humana Press.

Melo, O., y Vargas, R. (2003). Evaluación ecológica y silvicultural de ecosistemas boscosos. Universidad del Tolima CRQ, CARDER, CORPOCALDAS, CORTOLIMA. https://bit.ly/3uIC7jf

Morales S., León, y Varón P. T. (2006). Árboles ornamentales en el Valle del Aburrá, Elementos de manejo. Medellín, Colombia: Área Metropolitana del Valla del Aburrá.

Moreno, C. E. (2001). Métodos para medir la biodiversidad, Vol I. Zaragoza (España): CYTED, Programa Iberoamericano de Ciencia y Tecnología para el Desarrollo, ORCYT - UNESCO Oficina Regional de Ciencia y Tecnología para América Latina y el Caribe, UNESCO. Sociedad Entomológica Aragonesa (SEA).

Ozguner, H., y Kendle, A. D. (2006). Public attitudes towards naturalistic versus designed landscapes in the city of Sheffield (UK). Landscape and Urban Planning, 74(2), 139-157. https://doi.org/10.1016/j.landurbplan.2004.10.003

Palacio, A., y Pedraza, C. (2012). Inventario e identificación del componente arbóreo de la sede del Poblado Del Politécnico Jaime Isaza Cadavid. Revista Politécnica, 14(8): 85-95.

Padullés, J., Vila, J., y Barriocanal, C. (2015). Biodiversidad vegetal y ciudad: Aproximaciones desde la ecología urbana. Boletín de la Asociación de geógrafos españoles, 8, 83-107. http://dx.doi.org/10.21138/bage.1854

Qiu, L., y Nielsen, A. B. (2015). Are Perceived Sensory Dimensions a Reliable Tool for Urban Green Space Assessment and Planning? Landscape Research, 40(7), 834-854. https://doi.org/10.1080/01426397.2015.1029445

Salbitano, F., Simone, B., Michela, C. y Yujuan, C. (2017). Directrices para la silvicultura urbana y periurbana. Roma: FAO.

Sánchez, R. (2004). La observación participante como escenario y configuración de la diversidad de significados. En M. Torres (Ed.), Observar, escuchar y comprender. Sobre la tradición cualitativa en investigación social. México: PLACSO, El Colegio de México.

Schipperijn, J., Stigsdotter, U. K., Randrup, T. B., y Troelsen, J. (2010). Influences on the use of urban green space - A case study in Odense, Denmark. Urban Forestry \& Urban Greening, 9(1), 25-32. https://doi.org/10.1016/j.ufug.2009.09.002

Tovar Corso, G. (2013). Aproximación a la Silvicultura Urbana en Colombia. Bitácora Urbano Territorial, 22(1). https://bit.ly/3tlWZwh 
Wan, C., y Shen, G. Q. (2015). Encouraging the use of urban green space: The mediating role of attitude, perceived usefulness and perceived behavioural control. Habitat International, 50, 130-139. https://doi.org/10.1016/j.habitatint.2015.08.010

Wang, R., Zhao, J., y Liu, Z. (2016). Consensus in visual preferences: The effects of aesthetic quality and landscape types. Urban Forestry \& Urban Greening, 20, 210-217. https://doi.org/10.1016/j.ufug.2016.09.005

Williams, N. S. G., Schwartz, M. W., Vesk, P. A., Mccarthy, M. A., Hahs, A. K., Clematis, S. E., Corlett, R. T., Duncan, R. P., Norton, B. A., Thompson, K., y McdonnelL, M. J. (2009). A conceptual framework for predicting the effects of urban environments on flores. Journal of Ecology, 97(1) 4-9. https://doi.org/10.1111/j.1365-2745.2008.01460.x

Zhang, W., Yang, J., Ma, L., y Huang, C. (2015). Factors affecting the use of urban green spaces for physical activities: Views of young urban residents in Beijing. Urban Forestry \& Urban Greening, 14(4), 851-857. https://doi.org/10.1016/j.ufug.2015.08.006 
Anexo 1. Composición florística y diversidad vegetal presente para la flora urbana en el municipio de Peque.

\begin{tabular}{|c|c|c|c|c|c|}
\hline Familia & Especie & $\begin{array}{l}\text { Nombre } \\
\text { común }\end{array}$ & $\begin{array}{l}\text { Hábito de } \\
\text { crecimien } \\
\text { to }\end{array}$ & $\begin{array}{c}\text { Número de } \\
\text { géneros/Fa } \\
\text { milia }\end{array}$ & $\begin{array}{c}\text { Número de } \\
\text { especies/Fa } \\
\text { milia }\end{array}$ \\
\hline \multirow[t]{4}{*}{ Acanthaceae } & $\begin{array}{l}\text { Pachystachys lutea } \\
\text { Nees }\end{array}$ & $\begin{array}{l}\text { Camarón } \\
\text { amarillo }\end{array}$ & $\mathrm{AR}$ & \multirow[t]{4}{*}{4} & \multirow[t]{4}{*}{4} \\
\hline & $\begin{array}{l}\text { Trichanthera } \\
\text { gigantea (Bonpl) } \\
\text { Nees }\end{array}$ & $\begin{array}{l}\text { Quiebra } \\
\text { barrigo, } \\
\text { Nacedero }\end{array}$ & A & & \\
\hline & $\begin{array}{l}\text { Megaskepasma } \\
\text { erythrochlamys } \\
\text { Lindau }\end{array}$ & $\begin{array}{l}\text { Pluma de } \\
\text { Ángel }\end{array}$ & AR & & \\
\hline & $\begin{array}{l}\text { Ruellia simplex } \mathrm{C} . \\
\text { Wright }\end{array}$ & $\begin{array}{l}\text { Petunia } \\
\text { morada }\end{array}$ & $\mathrm{H}$ & & \\
\hline \multirow[t]{3}{*}{$\begin{array}{l}\text { Anacardiacea } \\
\text { e }\end{array}$} & $\begin{array}{l}\text { Anacardium } \\
\text { excelsum (Bert } \\
\text { \&Balb. Ex Kunth) } \\
\text { Skeels }\end{array}$ & $\begin{array}{l}\text { Caracolí, } \\
\text { Caracol, } \\
\text { Espavé }\end{array}$ & A & \multirow[t]{3}{*}{3} & \multirow[t]{3}{*}{3} \\
\hline & $\begin{array}{l}\text { Spondias purpurea } \\
\mathrm{L}\end{array}$ & Ciruela & AR & & \\
\hline & Mangifera indica $\mathrm{L}$ & $\begin{array}{l}\text { Mango, } \\
\text { Manga }\end{array}$ & A & & \\
\hline \multirow[t]{2}{*}{ Annonaceae } & Annona muricata $\mathrm{L}$ & $\begin{array}{l}\text { Guanábana, } \\
\text { Zapote } \\
\text { agrio, } \\
\text { Guanábano } \\
\text { y Catuche }\end{array}$ & A & \multirow[t]{2}{*}{1} & \multirow[t]{2}{*}{2} \\
\hline & Annona squamosa $\mathrm{L}$ & $\begin{array}{l}\text { Anón, } \\
\text { riñón, } \\
\text { anona, o } \\
\text { saramuyo }\end{array}$ & AR & & \\
\hline \multirow[t]{3}{*}{ Apocynaceae } & $\begin{array}{l}\text { Thevetia peruviana } \\
\text { (Pers.) K.Schum }\end{array}$ & $\begin{array}{l}\text { Cabalonga, } \\
\text { Catape }\end{array}$ & AR & \multirow[t]{3}{*}{2} & \multirow[t]{3}{*}{3} \\
\hline & $\begin{array}{l}\text { Thevetia ahouai }(\mathrm{L}) \\
\text { A.DC }\end{array}$ & $\begin{array}{l}\text { Huevo de } \\
\text { perro, } \\
\text { Campanilla }\end{array}$ & AR & & \\
\hline & $\begin{array}{l}\text { Catharanthus } \\
\text { roseus (L.) G.Don }\end{array}$ & Vinca & $\mathrm{H}$ & & \\
\hline \multirow[t]{3}{*}{ Araceae } & $\begin{array}{l}\text { Anthurium spp } \\
\text { Schott }\end{array}$ & Anturio & $\mathrm{H}$ & \multirow[t]{3}{*}{6} & \multirow[t]{3}{*}{7} \\
\hline & $\begin{array}{l}\text { Aglaonema } \\
\text { costatum N.E } \\
\text { Brown } \\
\end{array}$ & Dólar & $\mathrm{H}$ & & \\
\hline & $\begin{array}{l}\text { Aglaonema } \\
\text { conmutatum Schott }\end{array}$ & $\begin{array}{l}\text { Cafeto del } \\
\text { Brasil }\end{array}$ & $\mathrm{H}$ & & \\
\hline
\end{tabular}




\begin{tabular}{|c|c|c|c|c|c|}
\hline Familia & Especie & $\begin{array}{l}\text { Nombre } \\
\text { común }\end{array}$ & $\begin{array}{l}\text { Hábito de } \\
\text { crecimien } \\
\text { to }\end{array}$ & $\begin{array}{l}\text { Número de } \\
\text { géneros/Fa } \\
\text { milia }\end{array}$ & $\begin{array}{l}\text { Número de } \\
\text { especies/Fa } \\
\text { milia }\end{array}$ \\
\hline & $\begin{array}{l}\text { Caladium cf bicolor } \\
\text { (Aiton) Vent }\end{array}$ & $\begin{array}{l}\text { Corazón de } \\
\text { Jesús, } \\
\text { Orejas de } \\
\text { Elefante }\end{array}$ & $\mathrm{H}$ & & \\
\hline & $\begin{array}{l}\text { Spathiphyllum } \\
\text { wallisii Regel }\end{array}$ & $\begin{array}{l}\text { Espatifilo o } \\
\text { Garcita }\end{array}$ & $\mathrm{H}$ & & \\
\hline & $\begin{array}{l}\text { Epipremnum } \\
\text { Aureum } \\
\text { (Linden \& André) } \\
\text { G.S.Bunting }\end{array}$ & $\begin{array}{l}\text { Miami, } \\
\text { Potos }\end{array}$ & B & & \\
\hline & $\begin{array}{l}\text { Singonium c.f } \\
\text { podophyllum Schott }\end{array}$ & $\begin{array}{l}\text { Oreja de } \\
\text { Burro }\end{array}$ & $\mathrm{H}$ & & \\
\hline \multirow[t]{3}{*}{ Araliaceae } & $\begin{array}{l}\text { Polyscias guilfoylei } \\
\text { (W. Bull.) L.H. } \\
\text { Bayley }\end{array}$ & Millón & SU & \multirow[t]{3}{*}{2} & \multirow[t]{3}{*}{3} \\
\hline & $\begin{array}{l}\text { Polyscias } \\
\text { scutellaria (Burman } \\
\text { f) Fosberg }\end{array}$ & Millonaria & SU & & \\
\hline & $\begin{array}{l}\text { Schefflera } \\
\text { actinophylla (Endl) } \\
\text { Harms }\end{array}$ & Cheflera & AR & & \\
\hline $\begin{array}{l}\text { Araucariacea } \\
\mathrm{e}\end{array}$ & $\begin{array}{l}\text { Araucaria excelsa } \\
\mathrm{R} . \mathrm{Br}\end{array}$ & $\begin{array}{l}\text { Araucaria, } \\
\text { Araucaria } \\
\text { real }\end{array}$ & A & 1 & 1 \\
\hline \multirow[t]{3}{*}{$\begin{array}{l}\text { Arecaceae } \\
\text { (Palmae) }\end{array}$} & Cocos nucifera $\mathrm{L}$ & $\begin{array}{l}\text { Coco, } \\
\text { cocotero }\end{array}$ & PAM & \multirow[t]{3}{*}{3} & \multirow[t]{3}{*}{3} \\
\hline & $\begin{array}{l}\text { Dypsis lutencesns } \\
\text { (H.Wendl) Beentje } \\
\text { \& J.Dransf }\end{array}$ & $\begin{array}{l}\text { Areca, } \\
\text { palma de } \\
\text { frutos de } \\
\text { oro }\end{array}$ & PAM & & \\
\hline & $\begin{array}{l}\text { Veitchia merrillii } \\
\text { (Becc) H. Moore }\end{array}$ & $\begin{array}{l}\text { Palma } \\
\text { Manila, } \\
\text { Palma de } \\
\text { Navidad }\end{array}$ & PAM & & \\
\hline $\begin{array}{l}\text { Asphodelace } \\
\text { ae }\end{array}$ & $\begin{array}{l}\text { Aloe vera }(\mathrm{L}) \text { Burm } \\
\mathrm{f}\end{array}$ & $\begin{array}{l}\text { Penca de } \\
\text { sábila }\end{array}$ & $\mathrm{H}$ & 1 & 1 \\
\hline \multirow[t]{3}{*}{$\begin{array}{l}\text { Asteraceae } \\
\text { (compositae) }\end{array}$} & $\begin{array}{l}\text { Centratherum } \\
\text { punctatum Cass }\end{array}$ & $\begin{array}{l}\text { Azulita, } \\
\text { Rompe } \\
\text { Dolor }\end{array}$ & $\mathrm{H}$ & \multirow[t]{3}{*}{3} & \multirow[t]{3}{*}{3} \\
\hline & $\begin{array}{l}\text { Chrysanthemum } \\
\operatorname{spp} \mathrm{L}\end{array}$ & Crisantemo & $\mathrm{H}$ & & \\
\hline & Helianthus annus $\mathrm{L}$. & Girasol & $\mathrm{H}$ & & \\
\hline $\begin{array}{l}\text { Balsaminacea } \\
\text { e }\end{array}$ & $\begin{array}{l}\text { Impatiens } \\
\text { balsamina } \mathrm{L}\end{array}$ & Caracucho & $\mathrm{H}$ & 1 & 1 \\
\hline
\end{tabular}




\begin{tabular}{|c|c|c|c|c|c|}
\hline Familia & Especie & $\begin{array}{l}\text { Nombre } \\
\text { común }\end{array}$ & $\begin{array}{l}\text { Hábito de } \\
\text { crecimien } \\
\text { to }\end{array}$ & $\begin{array}{l}\text { Número de } \\
\text { géneros/Fa } \\
\text { milia }\end{array}$ & $\begin{array}{c}\text { Número de } \\
\text { especies/Fa } \\
\text { milia }\end{array}$ \\
\hline Bambusaceae & $\begin{array}{l}\text { Guadua } \\
\text { angustifolia kunth }\end{array}$ & Guadua & $\mathrm{HG}$ & 1 & 1 \\
\hline Begoniaceae & $\begin{array}{l}\text { Begonia } \\
\text { semperflorens L }\end{array}$ & Conchita & $\mathrm{H}$ & 1 & 1 \\
\hline \multirow[t]{5}{*}{ Bignoniaceae } & $\begin{array}{l}\text { Spathodea } \\
\text { campanulata Beaw }\end{array}$ & $\begin{array}{l}\text { Tulipán } \\
\text { Africano, } \\
\text { Miona }\end{array}$ & $\mathrm{A}$ & \multirow[t]{5}{*}{4} & \multirow[t]{5}{*}{5} \\
\hline & $\begin{array}{l}\text { Tabebuia } \\
\text { chrysantha (Jacq) } \\
\text { Nicholson }\end{array}$ & $\begin{array}{l}\text { Guayacán } \\
\text { amarillo, } \\
\text { Chicalá }\end{array}$ & A & & \\
\hline & $\begin{array}{l}\text { Tabebuia rosea } \\
\text { (Bertol) A.DC }\end{array}$ & $\begin{array}{l}\text { Guayacán } \\
\text { rosado }\end{array}$ & $\mathrm{A}$ & & \\
\hline & $\begin{array}{l}\text { Jacaranda } \\
\text { mimosifolia D.Don }\end{array}$ & Gualanday & A & & \\
\hline & $\begin{array}{l}\text { Pyrostegia venusta } \\
\text { (Ker Gawl) Miers }\end{array}$ & $\begin{array}{l}\text { Lluvia de } \\
\text { oro }\end{array}$ & $\mathrm{B}$ & & \\
\hline \multirow[t]{2}{*}{ Bombacaceae } & $\begin{array}{l}\text { Ceiba pentandra } \\
\text { (L) Gaertn }\end{array}$ & $\begin{array}{l}\text { Ceiba, } \\
\text { Bonga }\end{array}$ & A & \multirow[t]{2}{*}{2} & \multirow[t]{2}{*}{2} \\
\hline & $\begin{array}{l}\text { Pseudobombax } \\
\text { septenatum (Jacq) } \\
\text { Dugand }\end{array}$ & $\begin{array}{l}\text { Ceiba } \\
\text { Verde, } \\
\text { Bonga, } \\
\text { Ceiba } \\
\text { barrigona, } \\
\text { Majagua }\end{array}$ & $\mathrm{A}$ & & \\
\hline Boraginaceae & $\begin{array}{l}\text { Cordia alliodora } \\
\text { (Ruiz \& Pav.) } \\
\text { Cham }\end{array}$ & $\begin{array}{l}\text { Nogal } \\
\text { cafetero, } \\
\text { Nogal, } \\
\text { Moho, Mo, } \\
\text { Solera, } \\
\text { Vara de } \\
\text { humo }\end{array}$ & $\mathrm{A}$ & 1 & 1 \\
\hline Burseraceae & $\begin{array}{l}\text { Bursera simaruba } \\
\text { (L) Sarg }\end{array}$ & $\begin{array}{l}\text { Indio } \\
\text { desnudo, } \\
\text { Resbala } \\
\text { mono, } \\
\text { Carate }\end{array}$ & A & 1 & 1 \\
\hline Cannaceae & Canna indica $\mathrm{L}$ & $\begin{array}{l}\text { Achira,Cañ } \\
\text { a de la } \\
\text { india }\end{array}$ & $\mathrm{H}$ & 1 & 1 \\
\hline Caricaceae & Carica papaya $\mathrm{L}$ & Papaya & $\mathrm{HG}$ & 1 & 1 \\
\hline Cecropiaceae & Cecropia spp Loefl & Yarumo & $\mathrm{A}$ & 1 & 1 \\
\hline Clusiaceae & $\begin{array}{l}\text { Mammea } \\
\text { americana } \mathrm{L}\end{array}$ & Mamey & A & 1 & 1 \\
\hline
\end{tabular}




\begin{tabular}{|c|c|c|c|c|c|}
\hline Familia & Especie & $\begin{array}{l}\text { Nombre } \\
\text { común }\end{array}$ & $\begin{array}{l}\text { Hábito de } \\
\text { crecimien } \\
\text { to } \\
\end{array}$ & $\begin{array}{l}\text { Número de } \\
\text { géneros/Fa } \\
\text { milia } \\
\end{array}$ & $\begin{array}{c}\text { Número de } \\
\text { especies/Fa } \\
\text { milia }\end{array}$ \\
\hline \multirow[t]{2}{*}{$\begin{array}{l}\text { Combretacea } \\
\text { e }\end{array}$} & $\begin{array}{l}\text { Terminalia catappa } \\
\mathrm{L}\end{array}$ & Almendro & A & \multirow[t]{2}{*}{1} & \multirow[t]{2}{*}{2} \\
\hline & $\begin{array}{l}\text { Terminalia } \\
\text { ivorensis A. Chev }\end{array}$ & $\begin{array}{l}\text { Terminalia, } \\
\text { Framiré, } \\
\text { Idigbo, } \\
\text { Tirisio }\end{array}$ & A & & \\
\hline Crassulaceae & $\begin{array}{l}\text { Kalanchoe } \\
\text { glossfeldiana Poell } \\
\mathrm{n}\end{array}$ & Kalanchoe & $\mathrm{H}$ & 1 & 1 \\
\hline \multirow[t]{2}{*}{ Cupresaceae } & Thuja orientalis L & Pino Libro & AR & \multirow[t]{2}{*}{2} & \multirow[t]{2}{*}{2} \\
\hline & $\begin{array}{l}\text { Cupressus } \\
\text { sempervirens L }\end{array}$ & Pino ciprés & A & & \\
\hline Cycadaceae & $\begin{array}{l}\text { Cycas cf circinalis } \\
\mathrm{L}\end{array}$ & $\begin{array}{l}\text { Palma } \\
\text { Cica, } \\
\text { Palma sagú }\end{array}$ & $\mathrm{AR}$ & 1 & 1 \\
\hline $\begin{array}{l}\text { Cyclanthacea } \\
\text { e }\end{array}$ & $\begin{array}{l}\text { Cardulovica } \\
\text { palmata Ruiz \& } \\
\text { Pav. }\end{array}$ & Iraca & $\mathrm{HG}$ & 1 & 1 \\
\hline \multirow[t]{3}{*}{ Dracaenaceae } & $\begin{array}{l}\text { Dracaena fragrans } \\
\text { (L) Ker- Gawler var } \\
\text { massangeana }\end{array}$ & $\begin{array}{l}\text { Dracena, } \\
\text { árbol de la } \\
\text { felicidad }\end{array}$ & $\mathrm{HG}$ & \multirow[t]{3}{*}{3} & \multirow[t]{3}{*}{3} \\
\hline & $\begin{array}{l}\text { Sansevieria } \\
\text { trifasciata Prain. }\end{array}$ & $\begin{array}{l}\text { Lengua de } \\
\text { suegra, } \\
\text { Mapaná }\end{array}$ & $\mathrm{H}$ & & \\
\hline & $\begin{array}{l}\text { Cordyline rubra } \\
\text { (Otto \& A.Dietr) } \\
\text { Kuntze }\end{array}$ & $\begin{array}{l}\text { Palma } \\
\text { carey, } \\
\text { Dracena } \\
\text { Roja } \\
\end{array}$ & $\mathrm{H}$ & & \\
\hline \multirow[t]{4}{*}{$\begin{array}{l}\text { Euphorbiacea } \\
\text { e }\end{array}$} & $\begin{array}{l}\text { Codiaeum } \\
\text { variegatum } \mathrm{L}\end{array}$ & Croto & $\mathrm{SU}$ & \multirow[t]{4}{*}{3} & \multirow[t]{4}{*}{4} \\
\hline & $\begin{array}{l}\text { Euphorbia milii Des } \\
\text { Moul }\end{array}$ & $\begin{array}{l}\text { Espina de } \\
\text { cristo, } \\
\text { Corona de } \\
\text { cristo } \\
\end{array}$ & SU & & \\
\hline & $\begin{array}{l}\text { Euphorbia } \\
\text { pulcherrima Willd. } \\
\text { ex Klotzsch }\end{array}$ & $\begin{array}{l}\text { Lengua de } \\
\text { diablo }\end{array}$ & AR & & \\
\hline & $\begin{array}{l}\text { Acalypha } \\
\text { amentaceae f. } \\
\text { circinata } \\
\text { (Müll.Arg.) Fosberg }\end{array}$ & Acalifa & AR & & \\
\hline Fabaceae & $\begin{array}{l}\text { Erythrina crista- } \\
\text { galli } \mathrm{L}\end{array}$ & $\begin{array}{l}\text { Cresta de } \\
\text { gallo }\end{array}$ & AR & 8 & 10 \\
\hline
\end{tabular}




\begin{tabular}{|c|c|c|c|c|c|}
\hline Familia & Especie & $\begin{array}{l}\text { Nombre } \\
\text { común }\end{array}$ & $\begin{array}{l}\text { Hábito de } \\
\text { crecimien } \\
\text { to } \\
\end{array}$ & $\begin{array}{l}\text { Número de } \\
\text { géneros/Fa } \\
\text { milia }\end{array}$ & $\begin{array}{c}\text { Número de } \\
\text { especies/Fa } \\
\text { milia }\end{array}$ \\
\hline & $\begin{array}{l}\text { Eryhrina variegata } \\
\text { L. }\end{array}$ & $\begin{array}{l}\text { Búcaro } \\
\text { variegado: } \\
\text { Árbol del } \\
\text { coral Hindú }\end{array}$ & $\mathrm{A}$ & & \\
\hline & $\begin{array}{l}\text { Gliricidia sepium } \\
\text { (Jacq) kunth. Ex } \\
\text { walp }\end{array}$ & Matarratón & AR & & \\
\hline & $\begin{array}{l}\text { Acacia } \\
\text { mangium Willd }\end{array}$ & $\begin{array}{l}\text { Acacia } \\
\text { Mangium }\end{array}$ & AR & & \\
\hline & $\begin{array}{l}\text { Caesalpinia } \\
\text { pulcherrima }(L) \mathrm{Sw}\end{array}$ & $\begin{array}{l}\text { Clavellino, } \\
\text { Pequeño } \\
\text { Flamboyán }\end{array}$ & AR & & \\
\hline & $\begin{array}{l}\text { Arachis pintoi Krap } \\
\text { et Greg }\end{array}$ & $\begin{array}{l}\text { Maní } \\
\text { forrajero }\end{array}$ & $\mathrm{H}$ & & \\
\hline & $\begin{array}{l}\text { Senna spectabilis } \\
\text { (D.C) H.S Irwin } \\
\text { \&Barneby }\end{array}$ & $\begin{array}{l}\text { Velero, } \\
\text { caña fístulo } \\
\text { macho }\end{array}$ & $\mathrm{A}$ & & \\
\hline & $\begin{array}{l}\text { Senna cf gardneri } \\
\text { (Benth.) H.S. Irwin } \\
\text { \& Barneby }\end{array}$ & Velero & AR & & \\
\hline & $\begin{array}{l}\text { Albizia } \\
\text { guachapele (Kunth) } \\
\text { Dugand }\end{array}$ & $\begin{array}{l}\text { Cedro } \\
\text { Amarillo }\end{array}$ & A & & \\
\hline & $\begin{array}{l}\text { Enterolobium } \\
\text { cyclocarpum (Jacq.) } \\
\text { Griseb }\end{array}$ & $\begin{array}{l}\text { Piñón de } \\
\text { oreja }\end{array}$ & $\mathrm{A}$ & & \\
\hline \multirow[t]{2}{*}{ Geraniaceae } & $\begin{array}{l}\text { Pelargonium } \\
\text { spp_L'Hér. }\end{array}$ & Novios & $\mathrm{H}$ & 1 & 1 \\
\hline & Geranium spp L & Geranios & $\mathrm{H}$ & 1 & 1 \\
\hline $\begin{array}{l}\text { Heliconiacea } \\
\mathrm{e}\end{array}$ & Heliconia spp L & Heliconia & HG & 1 & 1 \\
\hline $\begin{array}{l}\text { Hydrangeace } \\
\text { ae }\end{array}$ & $\begin{array}{l}\text { Hydrangea } \\
\text { macrophylla } \\
\text { (Thunb) Ser } \\
\end{array}$ & Hortensia & $\mathrm{H}$ & 1 & 1 \\
\hline $\begin{array}{l}\text { Hypoxidacea } \\
\text { e }\end{array}$ & $\begin{array}{l}\text { Curculigo } \\
\text { capitulata (Lour.) } \\
\text { Kuntze }\end{array}$ & Cúrculigo & $\mathrm{HG}$ & 1 & 1 \\
\hline \multirow[t]{2}{*}{$\begin{array}{l}\text { Lamiaceae } \\
\text { (labiatae) }\end{array}$} & Coleus blumei & $\begin{array}{l}\text { Coleo, } \\
\text { Gitana }\end{array}$ & $\mathrm{H}$ & \multirow[t]{2}{*}{2} & \multirow[t]{2}{*}{2} \\
\hline & $\begin{array}{l}\text { Salvia cf splendens } \\
\text { Sellow ex Schult. }\end{array}$ & Salvia & $\mathrm{H}$ & & \\
\hline
\end{tabular}




\begin{tabular}{|c|c|c|c|c|c|}
\hline Familia & Especie & $\begin{array}{l}\text { Nombre } \\
\text { común }\end{array}$ & $\begin{array}{l}\text { Hábito de } \\
\text { crecimien } \\
\text { to } \\
\end{array}$ & $\begin{array}{l}\text { Número de } \\
\text { géneros/Fa } \\
\text { milia }\end{array}$ & $\begin{array}{c}\text { Número de } \\
\text { especies/Fa } \\
\text { milia }\end{array}$ \\
\hline \multirow[t]{2}{*}{ Lauraceae } & $\begin{array}{l}\text { Persea americana } \\
\text { Mill }\end{array}$ & Aguacate & A & \multirow[t]{2}{*}{1} & \multirow[t]{2}{*}{2} \\
\hline & $\begin{array}{l}\text { Persea caerulea } \\
(\mathrm{R} \& \mathrm{P}) \mathrm{Mez}\end{array}$ & Aguacatillo & A & & \\
\hline \multirow[t]{2}{*}{ Liliaceae } & $\begin{array}{l}\text { Chlorophytum } \\
\text { comosum (Thunb) } \\
\text { Jacques }\end{array}$ & $\begin{array}{l}\text { Cinta } \\
\text { blanca }\end{array}$ & $\mathrm{H}$ & \multirow[t]{2}{*}{2} & \multirow[t]{2}{*}{2} \\
\hline & Lilium spp L & Lirio Rojo & $\mathrm{H}$ & & \\
\hline Lythraceae & $\begin{array}{l}\text { Lagerstroemia } \\
\text { indica (L) Pers. }\end{array}$ & $\begin{array}{l}\text { Árbol } \\
\text { Jupiter }\end{array}$ & AR & 1 & 1 \\
\hline \multirow[t]{2}{*}{ Malvaceae } & $\begin{array}{l}\text { Malvaviscus } \\
\text { penduliflorus (DC.) } \\
\text { Hadac }\end{array}$ & $\begin{array}{l}\text { Campanita } \\
\text { o San } \\
\text { Joaquín }\end{array}$ & AR & \multirow[t]{2}{*}{2} & \multirow[t]{2}{*}{2} \\
\hline & $\begin{array}{l}\text { Hibiscus rosa- } \\
\text { sinensis } \mathrm{L}\end{array}$ & $\begin{array}{l}\text { Hibisco, } \\
\text { Rosa de } \\
\text { China }\end{array}$ & AR & & \\
\hline Marantaceae & $\begin{array}{l}\text { Calathea spp. } \\
\text { G.Mey. }\end{array}$ & Calatea & $\mathrm{H}$ & 1 & 1 \\
\hline Meliaceae & Cedrella odorata L & Cedro & $\mathrm{A}$ & 1 & 1 \\
\hline \multirow[t]{2}{*}{ Moraceae } & $\begin{array}{l}\text { Artocarpus altilis } \\
\text { (Parkinson) Fosberg }\end{array}$ & $\begin{array}{l}\text { Árbol del } \\
\text { pan }\end{array}$ & $\mathrm{A}$ & \multirow[t]{2}{*}{2} & \multirow[t]{2}{*}{2} \\
\hline & Ficus benjamina $\mathrm{L}$ & $\begin{array}{l}\text { Caucho, } \\
\text { Laurel }\end{array}$ & $\mathrm{A}$ & & \\
\hline Musaceae & $\begin{array}{l}\text { Musa velutina } \\
\text { H.Wendl. \& Drude }\end{array}$ & Musa & $\mathrm{HG}$ & 1 & 1 \\
\hline \multirow[t]{2}{*}{ Myrtaceae } & $\begin{array}{l}\text { Eucalyptus spp } \\
\text { L'Hér. }\end{array}$ & Eucalipto & A & \multirow[t]{2}{*}{2} & \multirow[t]{2}{*}{2} \\
\hline & Eugenia uniflora & Eugenia & AR & & \\
\hline $\begin{array}{l}\text { Nyctaginacea } \\
\mathrm{e}\end{array}$ & $\begin{array}{l}\text { Bougainvillea } \\
\text { glabra Choisy }\end{array}$ & $\begin{array}{l}\text { Veranera, } \\
\text { Curazao }\end{array}$ & AR & 1 & 1 \\
\hline Oleaceae & $\begin{array}{l}\text { Fraxinus uhdei } \\
\text { (Wenz.) Lingelsh }\end{array}$ & Urapán & $\mathrm{A}$ & 1 & 1 \\
\hline Piperaceae & $\begin{array}{l}\text { Peperomia tritachia } \\
\text { Kunth }\end{array}$ & $\begin{array}{l}\text { Corazón de } \\
\text { hombre, } \\
\text { Cuartillo, } \\
\text { moneda }\end{array}$ & $\mathrm{H}$ & 1 & 1 \\
\hline Polypodaceae & $\begin{array}{l}\text { Platycerium } \\
\text { bifurcatum (Cav.) C } \\
\text {. Chr }\end{array}$ & Cuerno & $\mathrm{H}$ & 1 & 1 \\
\hline Rosaceae & Rosa spp L & Rosa & AR & 1 & 1 \\
\hline \multirow[t]{3}{*}{ Rubiaceae } & Coffea arábiga $\mathrm{L}$ & Café & AR & \multirow[t]{3}{*}{3} & \multirow[t]{3}{*}{3} \\
\hline & Ixora coccinea $\mathrm{L}$ & $\begin{array}{l}\text { Ixora Roja, } \\
\text { Coral }\end{array}$ & SU & & \\
\hline & $\begin{array}{l}\text { Mussaenda alicia } \\
\text { Hort. }\end{array}$ & Musaenda & $\mathrm{AR}$ & & \\
\hline
\end{tabular}




\begin{tabular}{|c|c|c|c|c|c|}
\hline Familia & Especie & $\begin{array}{l}\text { Nombre } \\
\text { común }\end{array}$ & $\begin{array}{c}\text { Hábito de } \\
\text { crecimien } \\
\text { to } \\
\end{array}$ & $\begin{array}{c}\text { Número de } \\
\text { géneros/Fa } \\
\text { milia }\end{array}$ & $\begin{array}{c}\text { Número de } \\
\text { especies/Fa } \\
\text { milia }\end{array}$ \\
\hline Rutaceae & $\begin{array}{l}\text { Swinglea glutinosa } \\
\text { (Blanco) Merr }\end{array}$ & Swinglea & AR & 1 & 1 \\
\hline \multirow[t]{2}{*}{ Sapindaceae } & $\begin{array}{l}\text { Sapindus saponaria } \\
\mathrm{L}\end{array}$ & Chumbinbo & A & \multirow[t]{2}{*}{2} & \multirow[t]{2}{*}{2} \\
\hline & $\begin{array}{l}\text { Melicocca bijuga } \\
\text { (Jacq) L }\end{array}$ & $\begin{array}{l}\text { Mamoncill } \\
\text { o, Mamón }\end{array}$ & A & & \\
\hline Solanaceae & $\begin{array}{l}\text { Cestrum nocturnum } \\
\mathrm{L}\end{array}$ & $\begin{array}{l}\text { Jazmín de } \\
\text { la noche, } \\
\text { Galán de la } \\
\text { noche }\end{array}$ & SU & 1 & 1 \\
\hline Sterculiaceae & $\begin{array}{l}\text { Guazuma ulmifolia } \\
\text { Lam }\end{array}$ & Guásimo & AR & 1 & 1 \\
\hline Verbenaceae & Duranta spp L & $\begin{array}{l}\text { Duranta, } \\
\text { Guarda } \\
\text { parques }\end{array}$ & AR & 1 & 1 \\
\hline Violaceae & Viola spp L & $\begin{array}{l}\text { Pensamient } \\
\text { o }\end{array}$ & $\mathrm{H}$ & 1 & 1 \\
\hline $\begin{array}{l}\text { Zingiberacea } \\
\text { e }\end{array}$ & $\begin{array}{l}\text { Alpinia purpurata } \\
\text { (Vieill.) K. Schum }\end{array}$ & Ginger rojo & $\mathrm{HG}$ & 1 & 1 \\
\hline
\end{tabular}

Fuente: Elaboración propia A: Árbol, (Plantas leñosas, con fuste definido y alturas mayores a 3 m), AR: Arbusto (Plantas leñosas, generalmente con fuste muy ramificado desde la base del tallo y alturas menores o iguales a $3 \mathrm{~m}$ ), ); SU: Subarbusto (Plantas ligeramente lignificadas en la base con fuste muy ramificado y alturas iguales o menores a $3 \mathrm{~m}$ );PAM: Palmas Arbóreas

Monoestipitadas (Palmas con estipe definido, y alturas mayores a $3 \mathrm{~m}$ ); H: Hierbas terrestres

(Plantas sin tejido leñoso, no trepadoras, que crecen directamente sobre el piso; HG: Hierba Gigante (Plantas con las mismas características del hábito $\mathrm{H}$, pero con alturas mayores de $3 \mathrm{~m}$ ). B: Bejuco (Planta trepadora con diámetro menor de $2 \mathrm{~cm}$ ),E: Epífita (Según Allaby, 1992; Alzate et al., 2013).

Anexo 2. Inventario de la flora urbana con fines de uso ornamental y paisajístico en el municipio de Peque.

\begin{tabular}{|c|l|c|c|c|c|c|}
\hline \multicolumn{1}{|c|}{ Familia } & \multicolumn{1}{|c|}{ Especie } & $\mathbf{n}_{\mathbf{i}}$ & $\mathbf{P}_{\mathbf{i}=\mathbf{n}_{\mathbf{i}} / \mathbf{N}}$ & $\begin{array}{c}\text { Riqueza } \\
\mathbf{C . M}\end{array}$ & Shannon & $\begin{array}{c}\text { Índice de } \\
\text { Berger- } \\
\text { Parker }\end{array}$ \\
\hline Acanthaceae & $\begin{array}{l}\text { Pachystachys lutea } \\
\text { Nees }\end{array}$ & 2 & 0.0025 & 0.0186 & 0.0149 & \\
\hline
\end{tabular}




\begin{tabular}{|c|c|c|c|c|c|c|}
\hline Familia & Especie & $\mathbf{n}_{\mathbf{i}}$ & $\mathbf{P}_{\mathbf{i}=\mathbf{n}_{\mathbf{i}} / \mathbf{N}}$ & $\begin{array}{l}\text { Riqueza } \\
\text { C.M }\end{array}$ & Shannon & $\begin{array}{l}\text { Índice de } \\
\text { Berger- } \\
\text { Parker }\end{array}$ \\
\hline & $\begin{array}{l}\text { Trichanthera } \\
\text { gigantea (Bonpl) } \\
\text { Nees }\end{array}$ & 2 & 0.0025 & 0.0186 & 0.0149 & \\
\hline & $\begin{array}{l}\text { Megaskepasma } \\
\text { erythrochlamys } \\
\text { Lindau }\end{array}$ & 2 & 0.0025 & 0.0186 & 0.0149 & \\
\hline & $\begin{array}{l}\text { Ruellia simplex } \mathrm{C} \text {. } \\
\text { Wright }\end{array}$ & 4 & 0.0051 & 0.0373 & 0.0269 & \\
\hline \multirow[t]{3}{*}{$\begin{array}{l}\text { Anacardiacea } \\
\text { e }\end{array}$} & $\begin{array}{l}\text { Anacardium } \\
\text { excelsum (Bert } \\
\text { \&Balb. Ex Kunth) } \\
\text { Skeels }\end{array}$ & 1 & 0.0012 & 0.0093 & 0.0080 & \\
\hline & $\begin{array}{l}\text { Spondias purpurea } \\
\mathrm{L}\end{array}$ & 1 & 0.0012 & 0.0093 & 0.0080 & \\
\hline & Mangifera indica $\mathrm{L}$ & 5 & 0.0064 & 0.0467 & 0.0323 & \\
\hline \multirow[t]{2}{*}{ Annonaceae } & Annona muricata $\mathrm{L}$ & 4 & 0.0051 & 0.0373 & 0.0269 & \\
\hline & Annona squamosa L & 1 & 0.0012 & 0.0093 & 0.0080 & \\
\hline \multirow[t]{3}{*}{ Apocynaceae } & $\begin{array}{l}\text { Thevetia peruviana } \\
\text { (Pers.) K.Schum }\end{array}$ & 1 & 0.0012 & 0.0093 & 0.0080 & \\
\hline & $\begin{array}{l}\text { Thevetia ahouai (L) } \\
\text { A.DC }\end{array}$ & 1 & 0.0012 & 0.0093 & 0.0080 & \\
\hline & $\begin{array}{l}\text { Catharanthus } \\
\text { roseus }(\text { L.) G.Don }\end{array}$ & 3 & 0.0038 & 0.0280 & 0.0211 & \\
\hline \multirow[t]{7}{*}{ Araceae } & $\begin{array}{l}\text { Anthurium spp } \\
\text { Schott }\end{array}$ & 2 & 0.0025 & 0.0186 & 0.0149 & \\
\hline & $\begin{array}{l}\text { Aglaonema } \\
\text { costatum N.E } \\
\text { Brown } \\
\end{array}$ & 2 & 0.0025 & 0.0186 & 0.0149 & \\
\hline & $\begin{array}{l}\text { Aglaonema } \\
\text { conmutatum Schott }\end{array}$ & 1 & 0.0012 & 0.0093 & 0.0080 & \\
\hline & $\begin{array}{l}\text { Caladium cf bicolor } \\
\text { (Aiton) Vent }\end{array}$ & 1 & 0.0012 & 0.0093 & 0.0080 & \\
\hline & $\begin{array}{l}\text { Spathiphyllum } \\
\text { wallisii Regel }\end{array}$ & 1 & 0.0012 & 0.0093 & 0.0080 & \\
\hline & $\begin{array}{l}\text { Epipremnum } \\
\text { Aureum } \\
\text { (Linden \& André) } \\
\text { G.S.Bunting }\end{array}$ & 1 & 0.0012 & 0.0093 & 0.0080 & \\
\hline & $\begin{array}{l}\text { Singonium c.f } \\
\text { podophyllum Schott }\end{array}$ & 1 & 0.0012 & 0.0093 & 0.0080 & \\
\hline Araliaceae & $\begin{array}{l}\text { Polyscias guilfoylei } \\
\text { (W. Bull.) L.H. } \\
\text { Bayley }\end{array}$ & 1 & 0.0012 & 0.0093 & 0.0080 & \\
\hline
\end{tabular}




\begin{tabular}{|c|c|c|c|c|c|c|}
\hline Familia & Especie & $\mathbf{n}_{\mathbf{i}}$ & 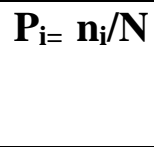 & $\begin{array}{c}\text { Riqueza } \\
\text { C.M }\end{array}$ & Shannon & $\begin{array}{c}\text { Índice de } \\
\text { Berger- } \\
\text { Parker }\end{array}$ \\
\hline & $\begin{array}{l}\text { Polyscias } \\
\text { scutellaria (Burman } \\
\text { f) Fosberg }\end{array}$ & 2 & 0.0025 & 0.0186 & 0.0149 & \\
\hline & $\begin{array}{l}\text { Schefflera } \\
\text { actinophylla (Endl) } \\
\text { Harms }\end{array}$ & 3 & 0.0038 & 0.0280 & 0.0211 & \\
\hline $\begin{array}{l}\text { Araucariacea } \\
\mathrm{e}\end{array}$ & $\begin{array}{l}\text { Araucaria excelsa } \\
\mathrm{R} . \mathrm{Br}\end{array}$ & 3 & 0.0038 & 0.0280 & 0.0211 & \\
\hline \multirow{3}{*}{$\begin{array}{l}\text { Arecaceae } \\
\text { (Palmae) }\end{array}$} & Cocos nucifera L & 3 & 0.0038 & 0.0280 & 0.0211 & \\
\hline & $\begin{array}{l}\text { Dypsis lutencesns } \\
\text { (H.Wendl) Beentje } \\
\text { \& J.Dransf }\end{array}$ & 3 & 0.0038 & 0.0280 & 0.0211 & \\
\hline & $\begin{array}{l}\text { Veitchia merrillii } \\
\text { (Becc) H. Moore }\end{array}$ & 16 & 0.0204 & 0.1495 & 0.0794 & \\
\hline \multirow[t]{3}{*}{$\begin{array}{l}\text { Asteraceae } \\
\text { (compositae) }\end{array}$} & $\begin{array}{l}\text { Centratherum } \\
\text { punctatum Cass }\end{array}$ & 2 & 0.0025 & 0.0186 & 0.0149 & \\
\hline & $\begin{array}{l}\text { Chrysanthemum spp } \\
\mathrm{L}\end{array}$ & 1 & 0.0012 & 0.0093 & 0.0080 & \\
\hline & Helianthus annus L. & 3 & 0.0038 & 0.0280 & 0.0211 & \\
\hline $\begin{array}{l}\text { Balsaminacea } \\
\mathrm{e}\end{array}$ & $\begin{array}{l}\text { Impatiens } \\
\text { balsamina } \mathrm{L}\end{array}$ & 2 & 0.0025 & 0.0186 & 0.0149 & \\
\hline Bambusaceae & $\begin{array}{l}\text { Guadua } \\
\text { angustifolia kunth }\end{array}$ & 9 & 0.0115 & 0.0841 & 0.0513 & \\
\hline Begoniaceae & $\begin{array}{l}\text { Begonia } \\
\text { semperflorens L }\end{array}$ & 1 & 0.0012 & 0.0093 & 0.0080 & \\
\hline \multirow[t]{5}{*}{ Bignoniaceae } & $\begin{array}{l}\text { Spathodea } \\
\text { campanulata Beaw }\end{array}$ & 4 & 0.0051 & 0.0373 & 0.0269 & \\
\hline & $\begin{array}{l}\text { Tabebuia } \\
\text { chrysantha (Jacq) } \\
\text { Nicholson }\end{array}$ & 5 & 0.0064 & 0.0467 & 0.0323 & \\
\hline & $\begin{array}{l}\text { Tabebuia rosea } \\
\text { (Bertol) A.DC }\end{array}$ & 1 & 0.0012 & 0.0093 & 0.0080 & \\
\hline & $\begin{array}{l}\text { Jacaranda } \\
\text { mimosifolia D.Don }\end{array}$ & 6 & 0.0076 & 0.0560 & 0.0370 & \\
\hline & $\begin{array}{l}\text { Pyrostegia venusta } \\
\text { (Ker Gawl) Miers }\end{array}$ & 2 & 0.0025 & 0.0186 & 0.0149 & \\
\hline \multirow[t]{2}{*}{ Bombacaceae } & $\begin{array}{l}\text { Ceiba pentandra } \\
\text { (L) Gaertn }\end{array}$ & 7 & 0.0089 & 0.0654 & 0.0420 & \\
\hline & $\begin{array}{l}\text { Pseudobombax } \\
\text { septenatum (Jacq) } \\
\text { Dugand }\end{array}$ & 2 & 0.0025 & 0.0186 & 0.0149 & \\
\hline
\end{tabular}




\begin{tabular}{|c|c|c|c|c|c|c|}
\hline Familia & Especie & $\mathbf{n}_{\mathbf{i}}$ & 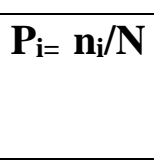 & $\begin{array}{c}\text { Riqueza } \\
\text { C.M }\end{array}$ & Shannon & $\begin{array}{c}\text { Índice de } \\
\text { Berger- } \\
\text { Parker }\end{array}$ \\
\hline Boraginaceae & $\begin{array}{l}\text { Cordia alliodora } \\
\text { (Ruiz \& Pav.) } \\
\text { Cham }\end{array}$ & 16 & 0.0204 & 0.1495 & 0.1101 & \\
\hline Burseraceae & $\begin{array}{l}\text { Bursera simaruba } \\
\text { (L) Sarg }\end{array}$ & 5 & 0.0064 & 0.0467 & 0.0323 & \\
\hline Cannaceae & Canna indica $\mathrm{L}$ & 2 & 0.0025 & 0.0186 & 0.0149 & \\
\hline Caricaceae & Carica papaya $\mathrm{L}$ & 2 & 0.0025 & 0.0186 & 0.0149 & \\
\hline Cecropiaceae & Cecropia spp Loefl & 1 & 0.0012 & 0.0093 & 0.0080 & \\
\hline Clusiaceae & $\begin{array}{l}\text { Mammea } \\
\text { americana } \mathrm{L}\end{array}$ & 1 & 0.0012 & 0.0093 & 0.0080 & \\
\hline \multirow[t]{2}{*}{$\begin{array}{l}\text { Combretacea } \\
\text { e }\end{array}$} & $\begin{array}{l}\text { Terminalia catappa } \\
\mathrm{L}\end{array}$ & 4 & 0.0051 & 0.0373 & 0.0269 & \\
\hline & $\begin{array}{l}\text { Terminalia } \\
\text { ivorensis A. Chev }\end{array}$ & 2 & 0.0025 & 0.0186 & 0.0149 & \\
\hline Crassulaceae & $\begin{array}{l}\text { Kalanchoe } \\
\text { glossfeldiana Poell } \\
\mathrm{n}\end{array}$ & 2 & 0.0025 & 0.0186 & 0.0149 & \\
\hline \multirow[t]{2}{*}{ Cupresaceae } & Thuja orientalis L & 3 & 0.0038 & 0.0280 & 0.0211 & \\
\hline & $\begin{array}{l}\text { Cupressus } \\
\text { sempervirens } \mathrm{L}\end{array}$ & 6 & 0.0076 & 0.0560 & 0.0370 & \\
\hline Cycadaceae & $\begin{array}{l}\text { Cycas cf circinalis } \\
\mathrm{L}\end{array}$ & 2 & 0.0025 & 0.0186 & 0.0149 & \\
\hline $\begin{array}{l}\text { Cyclanthacea } \\
\text { e }\end{array}$ & $\begin{array}{l}\text { Cardulovica } \\
\text { palmata Ruiz \& } \\
\text { Pav. }\end{array}$ & 3 & 0.0038 & 0.0280 & 0.0211 & \\
\hline \multirow[t]{3}{*}{ Dracaenaceae } & $\begin{array}{l}\text { Dracaena fragrans } \\
\text { (L) Ker- Gawler var } \\
\text { massangeana }\end{array}$ & 2 & 0.0025 & 0.0186 & 0.0149 & \\
\hline & $\begin{array}{l}\text { Sansevieria } \\
\text { trifasciata } \text { Prain. }\end{array}$ & 2 & 0.0025 & 0.0186 & 0.0149 & \\
\hline & $\begin{array}{l}\text { Cordyline rubra } \\
\text { (Otto \& A.Dietr) } \\
\text { Kuntze }\end{array}$ & 3 & 0.0038 & 0.0280 & 0.0211 & \\
\hline \multirow[t]{4}{*}{$\begin{array}{l}\text { Euphorbiacea } \\
\text { e }\end{array}$} & $\begin{array}{l}\text { Codiaeum } \\
\text { variegatum } \mathrm{L}\end{array}$ & 3 & 0.0038 & 0.0280 & 0.0211 & \\
\hline & $\begin{array}{l}\text { Euphorbia milii Des } \\
\text { Moul }\end{array}$ & 3 & 0.0038 & 0.0280 & 0.0211 & \\
\hline & $\begin{array}{l}\text { Euphorbia } \\
\text { pulcherrima Willd. } \\
\text { ex Klotzsch }\end{array}$ & 2 & 0.0025 & 0.0186 & 0.0149 & \\
\hline & $\begin{array}{l}\text { Acalypha } \\
\text { amentaceae }\end{array}$ & 12 & 0.0153 & 0.1121 & 0.0639 & \\
\hline Fabaceae & $\begin{array}{l}\text { Erythrina crista- } \\
\text { galli } \mathrm{L}\end{array}$ & 2 & 0.0025 & 0.0186 & 0.0149 & \\
\hline
\end{tabular}




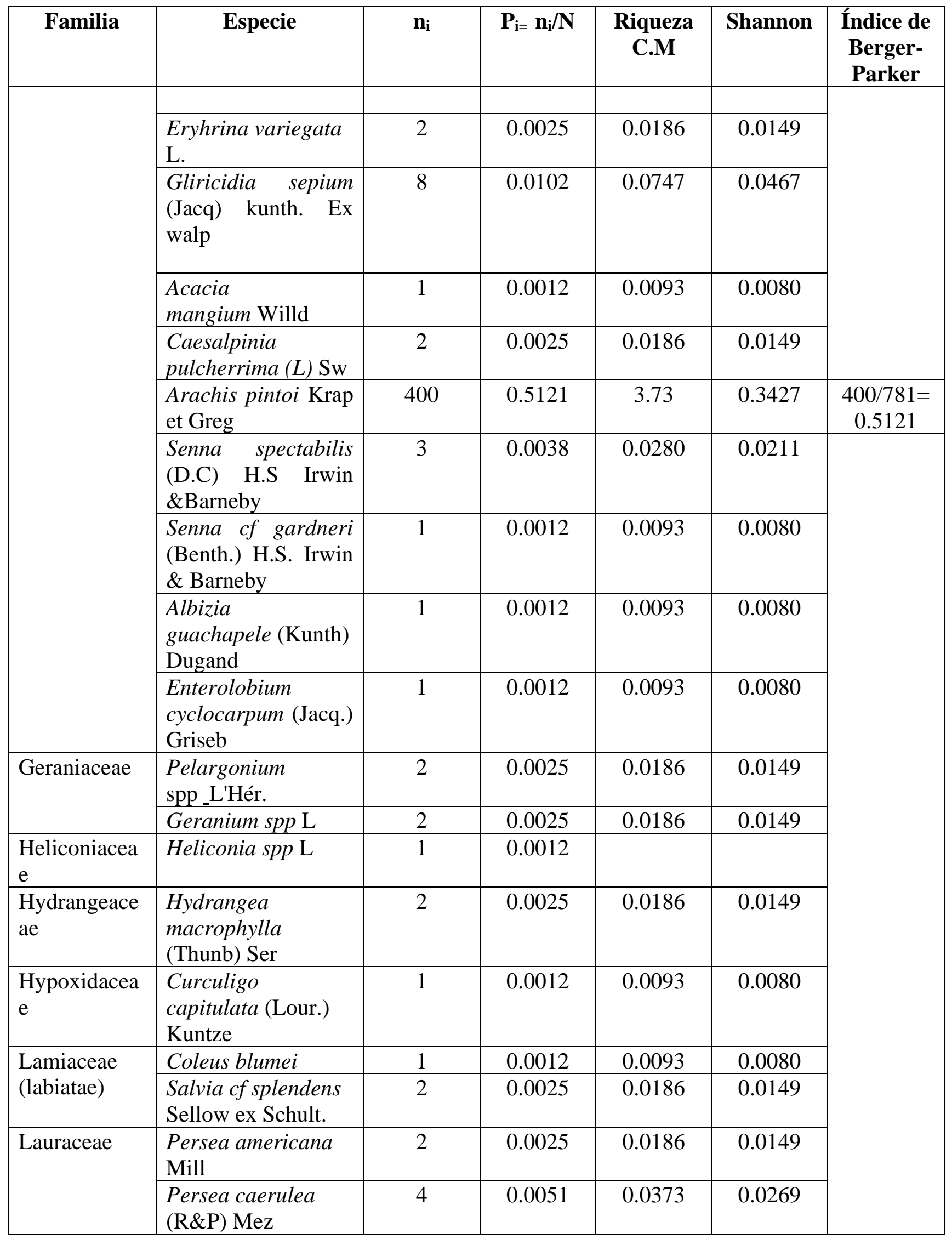




\begin{tabular}{|c|c|c|c|c|c|c|}
\hline Familia & Especie & $\mathbf{n}_{\mathbf{i}}$ & 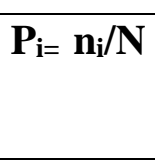 & $\begin{array}{c}\text { Riqueza } \\
\text { C.M }\end{array}$ & Shannon & $\begin{array}{c}\text { Índice de } \\
\text { Berger- } \\
\text { Parker }\end{array}$ \\
\hline \multirow[t]{3}{*}{ Liliaceae } & $\begin{array}{l}\text { Chlorophytum } \\
\text { comosum (Thunb) } \\
\text { Jacques }\end{array}$ & 2 & 0.0025 & 0.0186 & 0.0149 & \\
\hline & Lilium spp L & 2 & 0.0025 & 0.0186 & 0.0149 & \\
\hline & $\begin{array}{l}\text { Aloe vera }(\mathrm{L}) \\
\text { Burman f }\end{array}$ & 3 & 0.0038 & 0.0280 & 0.0211 & \\
\hline Lythraceae & $\begin{array}{l}\text { Lagerstroemia } \\
\text { indica }(\mathrm{L}) \text { Pers. }\end{array}$ & 6 & 0.0076 & 0.0560 & 0.0370 & \\
\hline \multirow[t]{2}{*}{ Malvaceae } & $\begin{array}{l}\text { Malvaviscus } \\
\text { penduliflorus (DC.) } \\
\text { Hadac }\end{array}$ & 2 & 0.0025 & 0.0186 & 0.0149 & \\
\hline & $\begin{array}{l}\text { Hibiscus rosa- } \\
\text { sinensis } \mathrm{L}\end{array}$ & 3 & 0.0038 & 0.0280 & 0.0211 & \\
\hline Marantaceae & $\begin{array}{l}\text { Calathea spp. } \\
\text { G.Mey. }\end{array}$ & 1 & 0.0012 & 0.0093 & 0.0080 & \\
\hline Meliaceae & Cedrella odorata $\mathrm{L}$ & 3 & 0.0038 & 0.0280 & 0.0211 & \\
\hline \multirow[t]{2}{*}{ Moraceae } & $\begin{array}{l}\text { Artocarpus altilis } \\
\text { (Parkinson) Fosberg }\end{array}$ & 1 & 0.0012 & 0.0093 & 0.0080 & \\
\hline & Ficus benjamina L & 2 & 0.0025 & 0.0186 & 0.0149 & \\
\hline Musaceae & $\begin{array}{l}\text { Musa velutina } \\
\text { H.Wendl. \& Drude }\end{array}$ & 1 & 0.0012 & 0.0093 & 0.0080 & \\
\hline \multirow[t]{2}{*}{ Myrtaceae } & $\begin{array}{l}\text { Eucalyptus spp } \\
\text { L'Hér. }\end{array}$ & 1 & 0.0012 & 0.0093 & 0.0080 & \\
\hline & Eugenia uniflora & 1 & 0.0012 & 0.0093 & 0.0080 & \\
\hline $\begin{array}{l}\text { Nyctaginacea } \\
\mathrm{e}\end{array}$ & $\begin{array}{l}\text { Bougainvillea } \\
\text { glabra Choisy }\end{array}$ & 3 & 0.0038 & 0.0280 & 0.0211 & \\
\hline Oleaceae & $\begin{array}{l}\text { Fraxinus uhdei } \\
\text { (Wenz.) Lingelsh }\end{array}$ & 1 & 0.0012 & 0.0093 & 0.0080 & \\
\hline Piperaceae & $\begin{array}{l}\text { Peperomia tritachia } \\
\text { Kunth }\end{array}$ & 1 & 0.0012 & 0.0093 & 0.0080 & \\
\hline Polypodaceae & $\begin{array}{l}\text { Platycerium } \\
\text { bifurcatum (Cav.) C } \\
\text {. Chr }\end{array}$ & 1 & 0.0012 & 0.0093 & 0.0080 & \\
\hline Rosaceae & Rosa spp L & 2 & 0.0025 & 0.0186 & 0.0149 & \\
\hline \multirow[t]{3}{*}{ Rubiaceae } & Coffea arábiga $\mathrm{L}$ & 1 & 0.0012 & 0.0093 & 0.0080 & \\
\hline & Ixora coccinea $\mathrm{L}$ & 2 & 0.0025 & 0.0186 & 0.0149 & \\
\hline & $\begin{array}{l}\text { Mussaenda alicia } \\
\text { Hort. }\end{array}$ & 1 & 0.0012 & 0.0093 & 0.0080 & \\
\hline Rutaceae & $\begin{array}{l}\text { Swinglea glutinosa } \\
\text { (Blanco) Merr }\end{array}$ & 25 & 0.0320 & 0.2336 & 0.1101 & \\
\hline \multirow[t]{2}{*}{ Sapindaceae } & $\begin{array}{l}\text { Sapindus saponaria } \\
\mathrm{L}\end{array}$ & 2 & 0.0025 & 0.0186 & 0.0149 & \\
\hline & $\begin{array}{l}\text { Melicocca bijuga } \\
\text { (Jacq) L }\end{array}$ & 2 & 0.0025 & 0.0186 & 0.0149 & \\
\hline
\end{tabular}




\begin{tabular}{|l|l|c|c|c|c|c|}
\hline \multicolumn{1}{|c|}{ Familia } & \multicolumn{1}{|c|}{ Especie } & $\mathbf{n}_{\mathbf{i}}$ & $\mathbf{P}_{\mathbf{i}=\mathbf{n}_{\mathbf{i}} / \mathbf{N}}$ & $\begin{array}{c}\text { Riqueza } \\
\mathbf{C . M}\end{array}$ & Shannon & $\begin{array}{c}\text { Índice de } \\
\text { Berger- } \\
\text { Parker }\end{array}$ \\
\hline Solanaceae & $\begin{array}{l}\text { Cestrum nocturnum } \\
\text { L }\end{array}$ & 1 & 0.0012 & 0.0093 & 0.0080 & \\
\hline Sterculiaceae & $\begin{array}{l}\text { Guazuma ulmifolia } \\
\text { Lam }\end{array}$ & 2 & 0.0025 & 0.0186 & 0.0149 \\
\hline Verbenaceae & Duranta spp L & 80 & 0.1024 & 0.7476 & 0.2333 \\
\hline Violaceae & Viola spp L & 2 & 0.0025 & 0.0186 & 0.0149 \\
\hline $\begin{array}{l}\text { Zingiberacea } \\
\text { e }\end{array}$ & $\begin{array}{l}\text { Alpinia purpurata } \\
\text { (Vieill.) K. Schum }\end{array}$ & 1 & 0.0012 & 0.0093 & 0.0080 \\
\hline Total & & 781 & & 7.2656 & 2.5917 \\
\hline
\end{tabular}

$\mathbf{n}_{\mathbf{i}}$ : Abundancia Absoluta $\mathbf{P}_{\mathbf{I}}$ : Abundancia Relativa o proporcional. C.M: Cociente de mezcla Fuente: Elaboración propia 